\title{
苯并氮杂环类荧光探针的设计、合成与应用研究进展
}

\author{
王 能 ${ }^{a} \quad$ Mani Arulkumar $^{a}$ 陈孝云*,b 王柏文 ${ }^{a}$ \\ 陈思鸿 ${ }^{a}$ 姚 辰 ${ }^{a}$ 汪朝阳*,a \\ ( ${ }^{a}$ 华南师范大学化学学院 教育部环境理论化学重点实验室 \\ 广州市生物医药分析化学重点实验室 广州 510006) \\ ( ${ }^{b}$ 江苏科技大学环境与化学工程学院 镇江 212003)
}

\begin{abstract}
摘要 苯并五元、六元氮杂环化合物具有刚性平面和大共轭结构, 能在多种有机溶剂、混合溶液中发出特征苂光, 且 结构中的 $\mathrm{N} 、 \mathrm{O} 、 \mathrm{~S}$ 杂原子可作为荧光探针的结合位点. 因此, 近年来苯并氮杂环化合物成为苂光探针领域研究焦点之 一. 从所用原料、合成方式、分子结构、作用机理等角度，重点介绍了苯并噁唑、苯并噻唑、苯并咪唑、吲哚及味唑 等苯并五元氮杂环，哇啉、苯并吡嗪、吩嗪等苯并六元氮杂环，以及两者共同构建的苯并多元氮杂环苂光探针的设计、 合成，并综述了它们对小分子、金属阳离子、阴离子、 $\mathrm{pH}$ 等多种分析物的检测应用.
\end{abstract}

关键词 苯并五元氮杂环; 苯并六元氮杂环; 苂光探针; 设计与合成; 识别机理；检测应用; 多功能化

\section{Research Progress in Design, Synthesis and Application of Benzo Nitrogen-Containing Heterocyclic Fluorescent Probes}

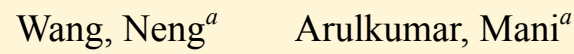 \\ Chen, Xiaoyun ${ }^{*, b}$ \\ Wang, Bowen ${ }^{a}$ \\ Chen, Sihong ${ }^{a} \quad$ Yao, Chen $^{a} \quad$ Wang, Zhaoyang ${ }^{*, a}$ \\ ( ${ }^{a}$ Key Laboratory of Theoretical Chemistry of Environment, Ministry of Education, Guangzhou Key Laboratory of Analytical \\ Chemistry for Biomedicine, School of Chemistry, South China Normal University, Guangzhou 510006) \\ ( ${ }^{b}$ School of Environmental and Chemical Engineering, Jiangsu University of Science and Technology, Zhenjiang 212003)
}

\begin{abstract}
Benzo five-/six-membered nitrogen-containing heterocyclic compound with a rigid plane and a large conjugate structure can emit characteristic fluorescence in a variety of organic solvents and mixed solutions, and $\mathrm{N}, \mathrm{O}, \mathrm{S}$ heteroatoms in the structure can serve as binding sites for fluorescent probes. Therefore, in recent years, benzo nitrogen-containing heterocyclic compounds are increasingly becoming one of the research focuses in the field of fluorescent probes. From the perspective of starting materials, synthesis methods, molecular structure, interaction mechanism, benzo five- / six-membered nitrogen-containing heterocyclic fluorescent probes containing the structure of benzoxazole, benzothiazole, benzimidazole, indole, carbazole, quinoline, benzopyrazine and phenazine are introduced with emphasis. And their detection application for a variety of analytes, such small molecules, metal cations, anions and $\mathrm{pH}$ are reviewed. In the future, it is worthy of further attention to the research on the integration of multiple heterocyclic functional structures into a multifunctional fluorescent probe by simple and green synthesis.

Keywords benzo five-membered nitrogen heterocycle; benzo six-membered nitrogen heterocycle; fluorescent probe; design and synthesis; recognition mechanism; detection application; multifunctionalization
\end{abstract}

苯并氮杂环类化合物由于具有较好的生物活性、多＼cjkstart变的结构以及良好的配位效应，在生物医药 ${ }^{[1]}$ 、催化材

\footnotetext{
* Corresponding authors. E-mail: wangzy@scnu.edu.cn; xiaoyun_chen12@163.com

Received April 25, 2019; revised May 14, 2019; published online June 3, 2019.

Project supported by the National Natural Science Foundation of China (No. 21602085), the Natural Science Foundation of Guangdong Province (No. 2014A030313429), the Guangzhou Science and Technology Project Scientific Special (No. 201607010251), the Natural Science Foundation of Jiangsu Province (No. BK20160551), the Guangdong Provincial Science and Technology Project (No. 2017A010103016), the Undergraduates Innovation Project of South China Normal University (No. 20191434).

国家自然科学基金(No. 21602085)、广东省自然科学基金(No. 2014A030313429)、广州市科技计划科学研究专项(No. 201607010251)、江苏省自然科 学基金(No. BK20160551)、广东省科技计划(No. 2017A010103016)、华南师范大学大创计划(No. 20191434)资助项目.
} 


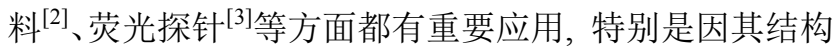
中含有可作为菼光团的大共轭结构和能与被检测物质 发生相互作用的氮原子, 近来在荧光传感器方面的应用 十分广泛 ${ }^{[4 ~ 6]}$. 鉴于此, 本文按照苯并五元氮杂环、苯并 六元氮杂环进行分类, 对苯并氮杂环类化合物作为苂光 探针的设计、合成与应用进行了综述.

\section{1 苯并五元氮杂环型荧光探针}

苯并五元氮杂环包含了苯并啞唑、苯并噻唑、苯并 咪唑、吲哚、咔唑等芳香化合物. 它们的结构中存在能 够吸收特征频率光的 $\mathrm{N}=\mathrm{N} 、 \mathrm{C}=\mathrm{N} 、 \mathrm{C}=\mathrm{C}$ 等发色团而 具有良好的荧光性能, 能够与离子、有机小分子、无机 分子等通过配位作用 ${ }^{[7 \sim 9]}$ 、氢键作用 ${ }^{[10]}$ 等改变自身的吸 收或苂光信号, 从而实现对分析物的传感.
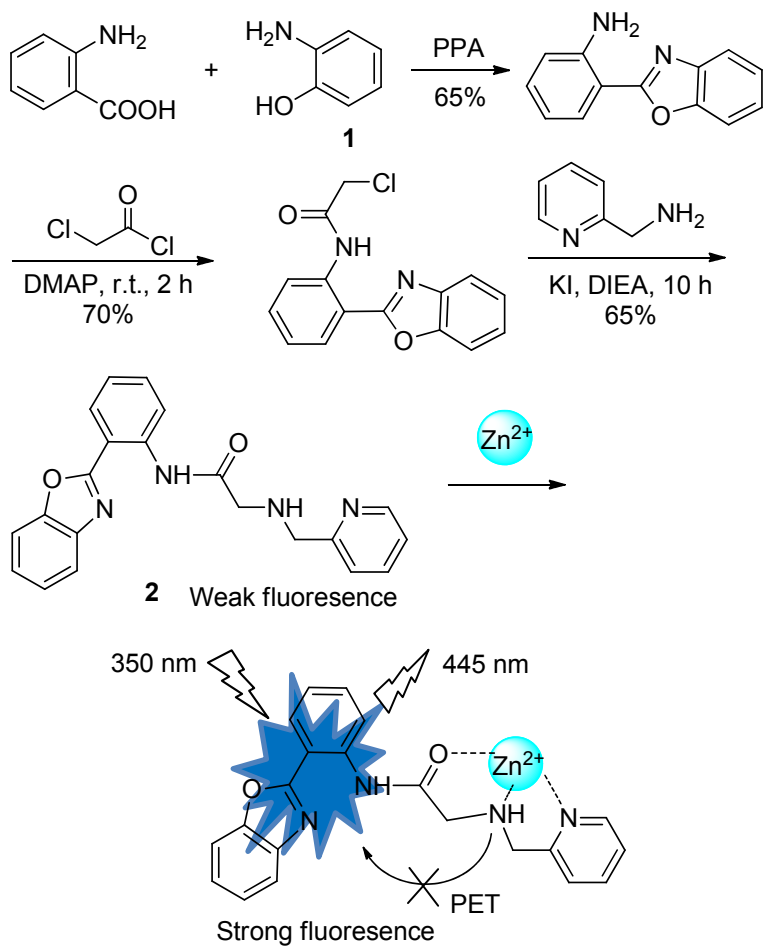

图式 1 探针 2 的合成与作用机理

Scheme 1 Synthesis of probe 2 and its interaction mechanism

\section{1 苯并噁唑类荧光探针}

苯并啞唑基团是同时含有 $\mathrm{O} 、 \mathrm{~N}$ 杂原子和大 $\pi$ 键的 芳杂环, 原料来源广, 合成相对简单. 其经典的合成是 以邻氨基苯酚与羧酸(或其衍生物)在强酸作用下脱水环 合生成. 例如郭炜课题组 ${ }^{[11]}$ 以邻氨基苯酚 1 和邻氨基苯 甲酸为原料, 在多聚磷酸(PPA)的催化下, 环合脱水构 建苯并噁唑环，再依次用碱 4-二甲氨基吡啶(DMAP)、

二异丙基乙胺(DIEA)催化, 经过酰胺化和亲核取代反 应，合成了一种用于 $\mathrm{Zn}^{2+}$ 检测的探针分子 2 (Scheme 1).
该探针分子以苯并噁唑为苂光团，2-氨甲基吡啶上的两 个氮原子以及酰胺上的氧原子与 $\mathrm{Zn}^{2+}$ 配位，使分子内 的光致电子转移(PET) 效应受到抑制, 金属配合物在 $445 \mathrm{~nm}$ 波长下苂光明显增强, 在 $\mathrm{pH}=5.2 \sim 12.1$ 范围内 实现对 $\mathrm{Zn}^{2+}$ 的 turn on 型检测.

类似地，张勇等 ${ }^{[12]}$ 以邻氨基苯酚 1 与对氨基苯甲酸 为原料, PPA 催化下先合成苯并噁唑, 再经二步反应合 成了一种对 $\mathrm{Hg}^{2+}$ 具有高选择性的 turn-off 型探针 $\mathbf{3}$ (Scheme 2). 不同于 turn-on 苂光增强型探针，该探针分 子本身由于苂光团苯并噁唑基的存在，在 $412 \mathrm{~nm}$ 波长 下有较强的荧光. 当分子内含孤对电子的 $\mathrm{N} 、 \mathrm{O} 、 \mathrm{~S}$ 原子 与具有空轨道的 $\mathrm{Hg}^{2+}$ 通过配位作用结合后, 苂光猝灭, 由蓝色变为无色.

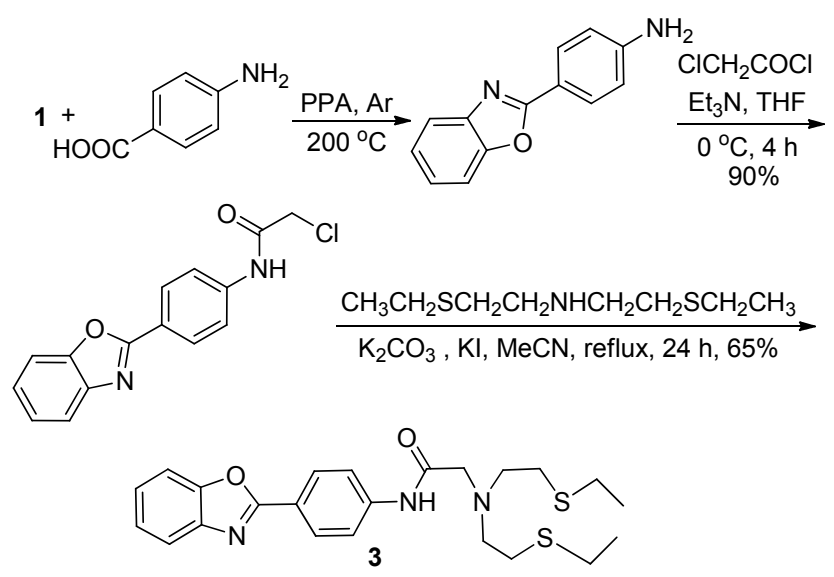

图式 2 探针 $\mathbf{3}$ 的合成

Scheme 2 Synthesis of probe 3

在国外, Yoon 课题组 ${ }^{[13]}$ 利用邻氨基苯酚、邻羟基苯 甲酸为原料，在 PPA 的作用下先合成苯并噁唑化合物 $\mathbf{4}$, 再经六亚甲基四胺(HMTA)引入醛基得到化合物 5, 5 进 一步与二氨基马来腈发生脱水缩合反应，最终得到了探 针 6 (Scheme 3). 起初由于二氨基马来腈中氨基强的 PET 效应，探针 6 在磷酸盐缓冲溶液中几乎无苂光，但 随着 $\mathrm{HClO}$ 的加入, $\mathrm{HClO}$ 诱导 $\mathrm{C}=\mathrm{N}$ 基团向 $\mathrm{CHO}$ 基团 转变, 致使其在 $440 \mathrm{~nm}$ 波长下荧光显著增强, 其他活性 物种如 $\mathrm{H}_{2} \mathrm{O}_{2} 、 \mathrm{NO} 、 \mathrm{ONOO}^{-}$等则对苂光无影响, 表明 6 对 $\mathrm{HClO}$ 具有较好的选择性，检测限达 $8 \times 10^{-8} \mathrm{~mol} \cdot \mathrm{L}^{-1}$.

陈卫华课题组 ${ }^{[14]}$ 利用化合物 $\mathbf{4}$ 与 HMAT 作用, 先制 备有 2 个醛基的化合物 7, 7 被还原得到以苯并噁唑为苂 光团的探针 8 (Scheme 4). 化合物 8 含有 2 个醇羟基，具 有很好的水溶性，可用于跟踪监测 $\mathrm{Zn}^{2+}$ 在植物生长运 输过程中的作用. 由于探针分子 $\mathbf{8}$ 自身的激发态分子内 质子转移(ESIPT)效应，在 $471 \mathrm{~nm}$ 有弱的浅蓝色苂光. 与 $\mathrm{Zn}^{2+}$ 配合后，ESIPT 过程被抑制，苂光发生蓝移，在 $451 \mathrm{~nm}$ 处有显著的亮蓝色苂光，苂光量子产率从 0.012 
增加至 0.131 , 且苂光强度在一定范围内随 $\mathrm{Zn}^{2+}$ 浓度的 增加呈线性增长, 故能实现对 $\mathrm{Zn}^{2+}$ 的定性定量检测.<smiles>O=C(O)c1ccccc1O</smiles><smiles>Oc1ccccc1-c1nc2ccccc2o1</smiles>
$\underset{50{ }^{\circ} \mathrm{C}}{\stackrel{\mathrm{HMTA}}{\longrightarrow}}$ $57 \%$<smiles>N#CC(=NC=Nc1ccc(O)c(-c2nc3ccccc3o2)c1)/C(N)=C(\N)C#N</smiles><smiles>C/C=C\C(=O)OC(O)c1ccc(O)c(-c2nc3ccccc3o2)c1</smiles>

图式 3 探针 $\mathbf{6}$ 的合成与作用机理

Scheme 3 Synthesis of probe 6 and its interaction mechanism
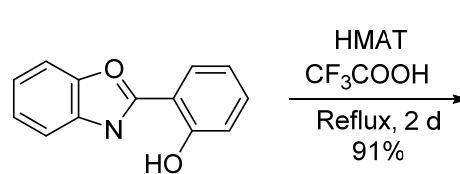

$91 \%$

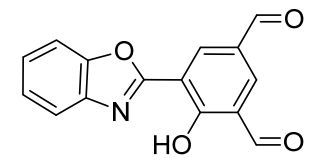

7

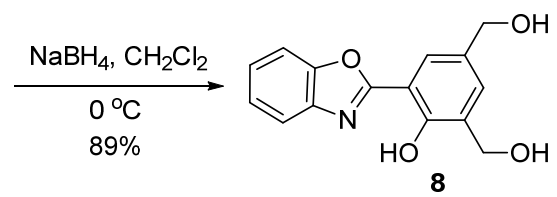

图式 4 探针 8 的合成

Scheme 4 Synthesis of probe 8

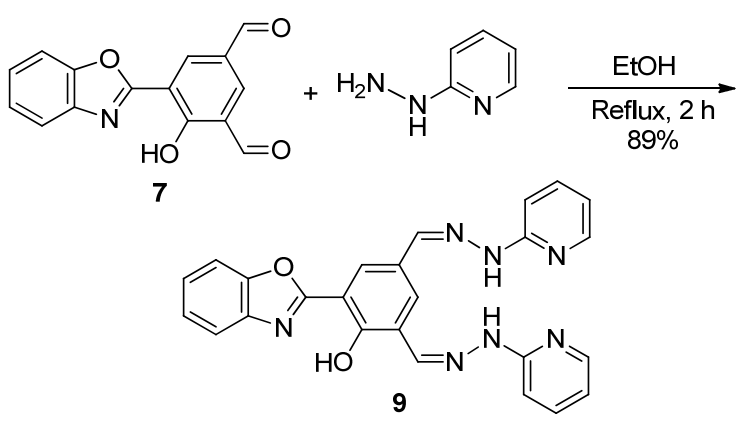

Pang 课题组 ${ }^{[15]}$ 则以化合物 7 为基础, 进一步与 2肼吡啶反应, 合成了能在水溶液同时检测金属离子 $\mathrm{Fe}^{3+} 、 \mathrm{Cr}^{3+} 、 \mathrm{Al}^{3+}$ 的探针分子 9 (Eq. 1). 有趣的是, 不同 于多数对 $\mathrm{Fe}^{3+} 、 \mathrm{Cr}^{3+}$ 的检测基于荧光猝灭原理, 该探针 对 $\mathrm{Fe}^{3+} 、 \mathrm{Cr}^{3+} 、 \mathrm{Al}^{3+}$ 的检测均是荧光增强型, 且对二价、
一价的金属离子无响应. 这是由于三价 $\mathrm{Fe}^{3+} 、 \mathrm{Cr}^{3+}$ 、 $\mathrm{Al}^{3+}$ 配位能力强于二价、一价金属离子, 能更好地与吡 啶环及亚胺键上的氮原子配位，并且由于两个吡啶肼间 的苯环上氢原子的空间位阻, $\mathrm{C}=\mathrm{N}$ 键与金属离子的配 位不发生在苯并啞唑环所处的平面, 这避免了 $\mathrm{C}=\mathrm{N}$ 键 对苂光的影响. 另外，与 $\mathrm{Fe}^{3+}$ 配合后的苂光明亮但持续 时间不长, 五分钟后便开始衰减, 而 $\mathrm{Cr}^{3+} 、 \mathrm{Al}^{3+}$ 配合物 的苂光较为稳定, 分别呈黄色、蓝绿色，故利用这一性 质可肉眼鉴别三种离子.

Pang 课题组 ${ }^{[16]}$ 还利用含两个羧基的二酸与邻氨基 苯酚缩合，制备了同时含两个苯并惡唑基的荧光探针 10 (Scheme 5). 利用同分异构体化合物 11 与其进行对比 研究, 发现由于空间结构的差异, 苯并啞唑基位于间位 的探针 10 更有利于产生 ESIPT 效应, 其与 $\mathrm{Zn}^{2+} 、 \mathrm{~F}^{-}$作 用时更明显地改变荧光信号，灵敏度更高，更合适于检 测 $\mathrm{Zn}^{2+}$ 和 $\mathrm{F}^{-[16,17]}$.<smiles>O=C(O)c1cc(C(=O)O)c(O)cc1O</smiles>

图式 5 探针 10 的合成及其同分异构体 $\mathbf{1 1}$ 的结构

Scheme 5 Synthesis of probe $\mathbf{1 0}$ and structure of isomer $\mathbf{1 1}$

另外，稠环芳香酸也可与邻氨基苯酚类化合物反 应，制备苯并噁唑型探针. 例如, Park 课题组 ${ }^{[18]}$ 以 3-差 基-2-菜酸和 3-甲基邻氨基苯酚为原料, 在 PPA 的催化 下先构建苯并噁唑环，再经多步反应合成了用于 $\mathrm{Zn}^{2+}$ 检测的 turn-on 型探针分子 $\mathbf{1 2}$ (Scheme 6). 其中，苯并噁 唑基既作苂光团同时又参与配位. 向探针 12 的 $\mathrm{MeCN}$<smiles>Cc1cccc(O)c1NC(=O)c1cc2ccccc2cc1-c1nc2c(C)cccc2o1</smiles><smiles>Oc1cc2ccccc2cc1-c1nc2c(CN(Cc3ccccn3)Cc3ccccn3)cccc2o1</smiles>

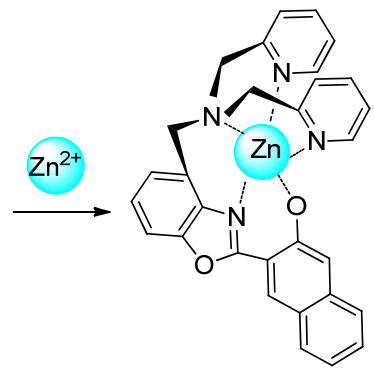

图式 6 探针 12 的合成与作用机理

Scheme 6 Synthesis of probe 12 and its interaction mechanism 
溶液中加入 $\mathrm{Zn}\left(\mathrm{ClO}_{4}\right)_{2}$ 时, 化合物 12 上所有 $\mathrm{N}$ 原子和芸 环羟基的 $\mathrm{O}$ 原子都与 $\mathrm{Zn}^{2+}$ 发生配位, 形成一个结合比为 $1: 1$ 且以 $\mathrm{Zn}^{2+}$ 为中心的扭曲三角双雉体. 由于 $\mathrm{Zn}^{2+}$ 的 配合作用, 致使探针分子 12 内部 PET 效应受到抑制, 且 菜环基团上羟基发生去质子化, 故荧光信号增强, 从而 实现对 $\mathrm{Zn}^{2+}$ 的选择性识别.

羧酸衍生物也能与邻氨基苯酚类化合物反应, 合成 苯并噁唑类荧光探针. 例如, Kikuchi 课题组 ${ }^{[19]}$ 用氧类化 合物和邻氨基苯酚衍生物反应, 合成了探针 $\mathbf{1 3}$ (Scheme 7), 其紫外吸收在不同 $\mathrm{pH}$ 范围表现出 off-on-off 型变化. 起初在 $\mathrm{pH}=4.8$ 时, 探针 $\mathbf{1 3}$ 在 $385 \mathrm{~nm}$ 处有最大紫外吸 收. 随着 $\mathrm{pH}$ 的增加, 由于羧酸的羟基与苯并噁唑上的 氮形成分子内氢键, 香豆素上的酚羟基首先发生去质子 化, 最大吸收峰红移至 $435 \mathrm{~nm}$ 处, 即 off-on 响应. 若 $\mathrm{pH}$ 继续增大, 羧基上的羟基也发生去质子化, 最大吸收峰 蓝移至 $400 \mathrm{~nm}$, 即 on-off 响应. 故可根据最大吸收峰的 变化实现对 $\mathrm{pH}$ 的可逆检测.
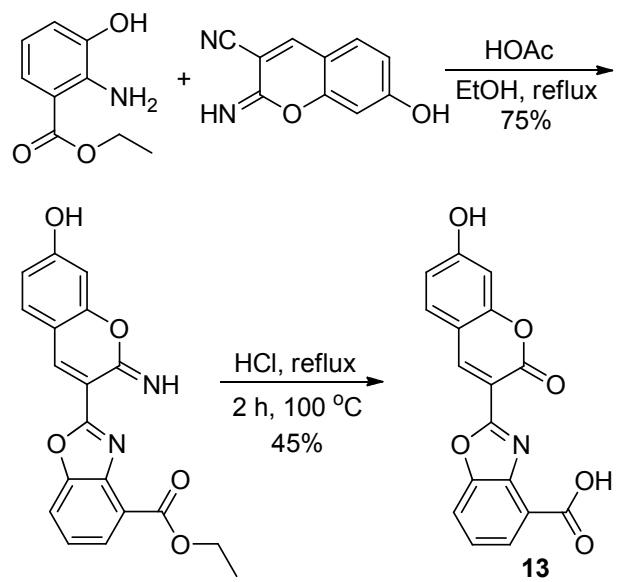

图式 7 探针 $\mathbf{1 3}$ 的合成

Scheme 7 Synthesis of probe 13

芳香醛与邻氨基酚类化合物间的反应也能合成苯 并噁唑类探针. 该过程一般是先通过脱水缩合反应生成 席夫碱, 再进一步氧化脱氢形成苯并噁唑环. 例如, 张 晓兵课题组 ${ }^{[20]}$ 用 2-羟基-4-烯丙氧基苯甲醛与邻氨基苯 酚先脱水反应, 再经 $\mathrm{BaMnO}_{4}$ 氧化脱氢, 合成了探针分 子 14 (Scheme 8). 由于探针 14 的末端有可发生聚合的 碳碳双键存在, 能以共价键的形式固定在改性石英玻璃 表面, 用于检测 $\mathrm{Zn}^{2+}$ 时, 同副族的 $\mathrm{Cd}^{2+}$ 对识别无影响. 由于 ESIPT 效应, 荧光发射峰位于 $450 \mathrm{~nm}$ 处. 当向溶液 中加入 $\mathrm{Zn}^{2+}$ 时, 由于锌离子的配位, ESIPT 过程被抑制, 荧光发生蓝移, 在 $404 \mathrm{~nm}$ 处发射荧光. 利用这一现象可 实现对 $\mathrm{Zn}^{2+}$ 传感, 检测限为 $4.0 \times 10^{-5} \mathrm{~mol} \cdot \mathrm{L}^{-1}$.

分子内同时含两个醛基的芳香醛也能与邻氨基苯 酚反应得到苯并噁唑. 例如, 杨小风课题组 ${ }^{[21]}$ 以邻羟基

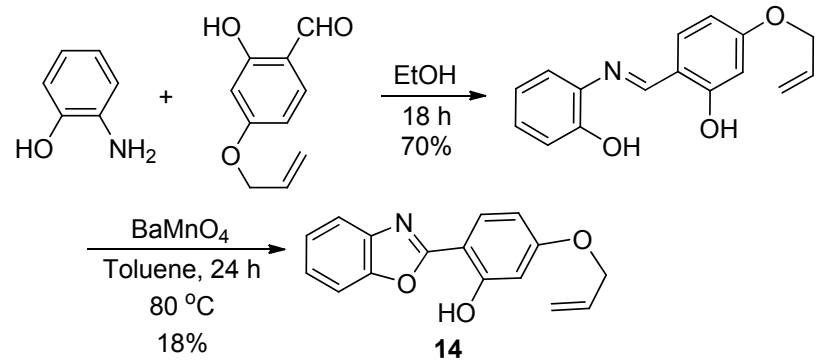

图式 8 探针 14 的合成

Scheme 8 Synthesis of probe 14

间苯二甲醛、邻氨基苯酚作原料, 先合成苯并噁唑化合 物 $\mathbf{1 5}$, 然后利用中间体 $\mathbf{1 5}$ 上的另一个醛基引入苂光团 罗丹明 B 衍生物, 合成了探针 $\mathbf{1 6}$ (Scheme 9). 基于键能 转移(TBET)效应, 在探针 $\mathbf{1 6}$ 的 $\mathrm{CH}_{3} \mathrm{CN} /$ Tris $(V / V=1 / 1$, $\mathrm{pH}=7.4$ )缓冲溶液中逐滴加入 $\mathrm{Hg}^{2+}$ 时, 其荧光信号呈比 率型及比色型变化, 并且配位后的苂光量子产率高达 0.64 , 检测限为 $1.3 \times 10^{-9} \mathrm{~mol} \cdot \mathrm{L}^{-1}$, 低于美国环保署对 饮用水中 $\mathrm{Hg}^{2+}$ 含量 $\left(2 \times 10^{-6} \mathrm{~mol} \cdot \mathrm{L}^{-} 1\right)$ 的要求, 能用于实 际水样检测.

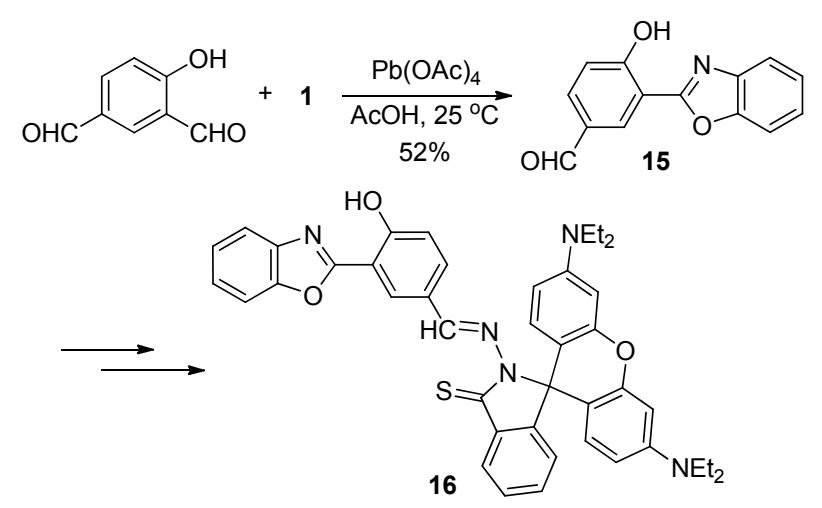

图式 9 探针 16 的合成

Scheme 9 Synthesis of probe 16

杂环芳香醛与邻氨基酚类化合物也能用于苯并噁 唑的合成. 例如, Costa 等 ${ }^{[22]}$ 报道了一系列含吡咯、咪唑 杂环基团的苯并噁唑-5-酰基丙氨酸衍生物的合成(以探 针 17 为例, Eq. 2), 它们在乙腈溶液中能很好地与 $\mathrm{Cu}^{2+}$ 、 $\mathrm{Pd}^{2+}$ 两种离子发生 $1: 2$ 配位, 且咪唑类衍生物的络合 强于吡咯类.

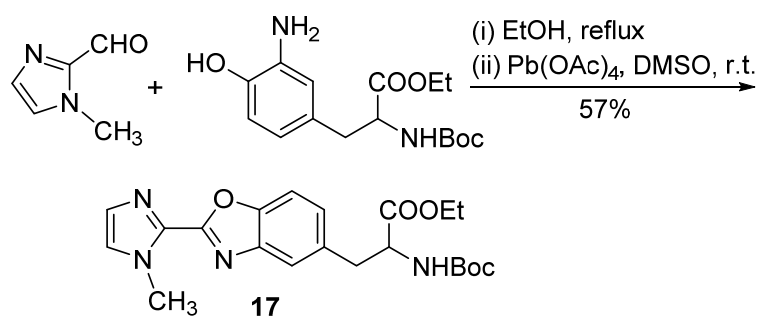




\section{2 苯并噻唑类荧光探针}

同样作为含氮杂环中的一员, 与苯并噁唑化合物相 似, 苯并噻唑类化合物由于分子所具有的共轭结构以及 硫、氮杂原子, 也具有良好的荧光性能 ${ }^{[23]}$. 实际上, 可 认为将苯并噁唑上的氧用硫原子替换, 即为苯并噻唑, 故其合成方法与苯并啞唑相似, 通常是用羧酸 ${ }^{[24]}$ 、醛 ${ }^{[25]}$ 或其衍生物与邻氨基苯硫酚反应制备.

Kaur 课题组 ${ }^{[26]}$ 以 4-氨基 2-羟基苯甲酸和邻氨基苯 硫酚 18 为原料, 在 PPA 催化下缩合得到苯并噻唑化合 物 19, 利用其中的氨基与对称的芳香醛化合物缩合, 可 得到探针分子 20 (Scheme 10). 探针 20 在 $\mathrm{Cu}^{2+}$ 存在时, 其 $\mathrm{C}=\mathrm{N}$ 双键发生水解, 生成化合物 19, 其中 $\mathrm{O}$ 和唑环 上 $\mathrm{N}$ 原子与 $\mathrm{Cu}^{2+}$ 发生配位, 形成金属配合物, 致使菼光 猝灭. 若继续向溶液中引入 $\mathrm{PO}_{4}^{3-}, \mathrm{Cu}^{2+}$ 将从金属配合 物中解脱, 转而与 $\mathrm{PO}_{4}^{3-}$ 形成更稳定的沉淀 $\mathrm{Cu}_{3}\left(\mathrm{PO}_{4}\right)_{2}$, 故荧光恢复. 基于此原理, 该探针可成功制备成便携式 传感纸条, 用于实际样品中 $\mathrm{Cu}^{2+}$ 的检测.

在国内，牛庆芬等 ${ }^{[27]}$ 用氭类化合物与 18 缩合反应，
再与含三个噻吩基团的醛缩合，制备了一种比色型、荧 光 turn on 型检测 $\mathrm{CN}^{-}$的探针 21 (Scheme 11). 在探针 21 溶液中滴加 $\mathrm{CN}^{-}$时, 可肉眼观测到溶液由橙色变为无 色, 荻光颜色由墨绿色变为蓝色. 这是由于 $\mathrm{CN}^{-}$与探针 21 中的 $\mathrm{C}=\mathrm{C}$ 双键发生亲核加成，致使由噻吩基团到苯 并噻唑环的分子内电荷转移(ICT) 效应抑制, 苂光得以 增强. 探针 21 对 $\mathrm{CN}^{-}$的检测限为 $4.6 \times 10^{-7} \mathrm{~mol} \cdot \mathrm{L}^{-1}$, 可 在食品样品分析检测 $\mathrm{CN}^{-}$, 用于海拉细胞的生物成像.

最近，曾文涁课题组 ${ }^{[28]}$ 用酸酐类化合物和 18 为原 料, 经多步反应, 制备了一种测 $\mathrm{Al}^{3+}$ 的探针 22 (Scheme 12). 该探针能自组装成几乎无荧光的纳米粒子, 在水 溶液中加入 $\mathrm{Al}^{3+}$ 时能进一步使探针 22 聚集, ESIPT 效应 加强, 在 $555 \mathrm{~nm}$ 下有显著的苂光增强现象, 其对 $\mathrm{Al}^{3+}$ 的检测限可达 $5.0 \times 10^{-10} \mathrm{~mol} \cdot \mathrm{L}^{-1}$.

醛类化合物也是合成苯并噻唑的常见原料 ${ }^{[29] ， 与 ~}$ 邻氨基苯硫酚缩合过程中, 通常有合适的氧化剂参与.

刘又年课题组 ${ }^{[30]}$ 用 5 -甲基邻差基苯甲醛与化合物 18 反应, 在合成苯并噻唑化合物 23 过程中, 使用了

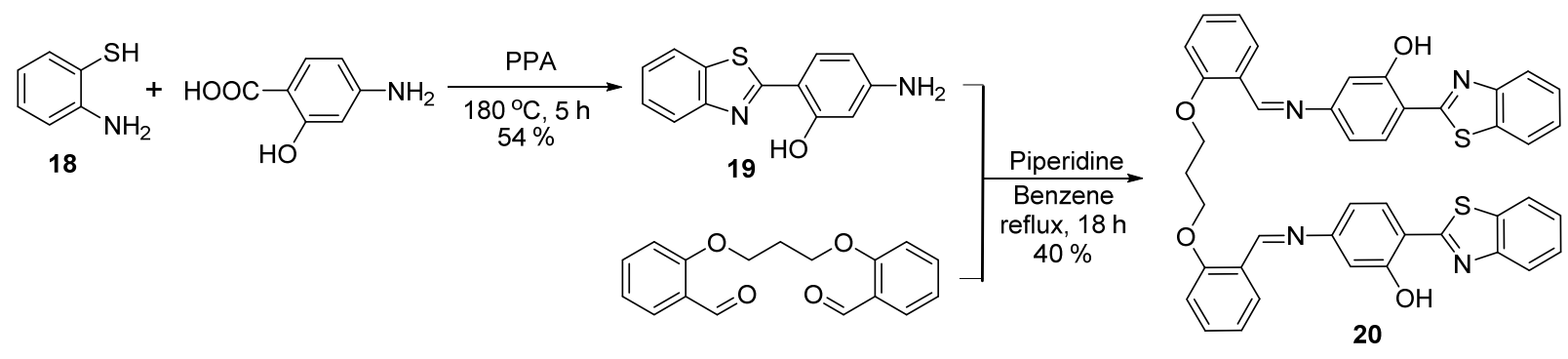

图式 10 探针 $\mathbf{2 0}$ 的合成

Scheme 10 Synthesis of probe 20

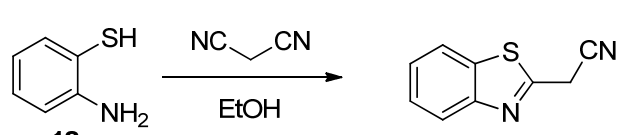

18

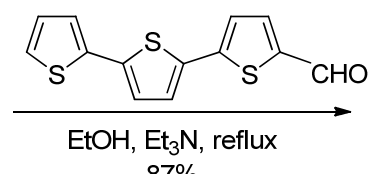

$87 \%$

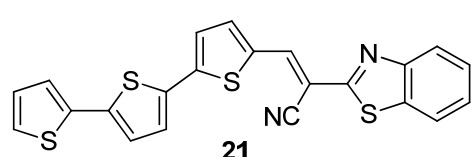

21

图式 11 探针 21 的合成

Scheme 11 Synthesis of probe $\mathbf{2 1}$

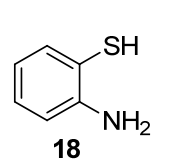<smiles>O=c1[nH]c2ccccc2c(=O)o1</smiles><smiles>CC(=O)OCc1ccccc1</smiles><smiles>Nc1ccccc1-c1nc2ccccc2s1</smiles>
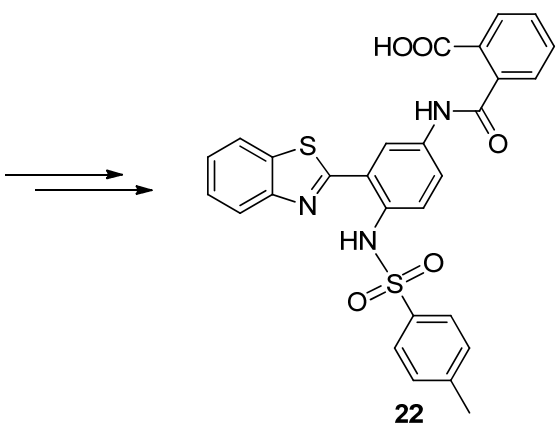

图式 12 探针 22 的合成

Scheme 12 Synthesis of probe 22 
氧化剂 $\mathrm{Na}_{2} \mathrm{~S}_{2} \mathrm{O}_{5}$. 接着引入醛基合成化合物 24, 继续衍 生化可合成探针 25 (Scheme 13). 探针 25 在 $350 \mathrm{~nm}$ 的 激发光下仅有微弱的苂光, 但在具有氧化性的 $\mathrm{ClO}^{-}$加 入后, 探针 $\mathbf{2 5}$ 的 $\mathrm{C}=\mathrm{N}$ 双键被氧化为强吸电子性的腈 基, 使荧光在 $540 \mathrm{~nm}$ 处显著增强, 斯托克斯位移达 190 $\mathrm{nm}$. 探针 25 对 $\mathrm{ClO}^{-}$的检测限达 $8.0 \times 10^{-7} \mathrm{~mol} \cdot \mathrm{L}^{-1}$, 且 因其具有很好的生物相容性和细胞通透性而可用于活 细胞中 $\mathrm{ClO}^{-}$的检测.

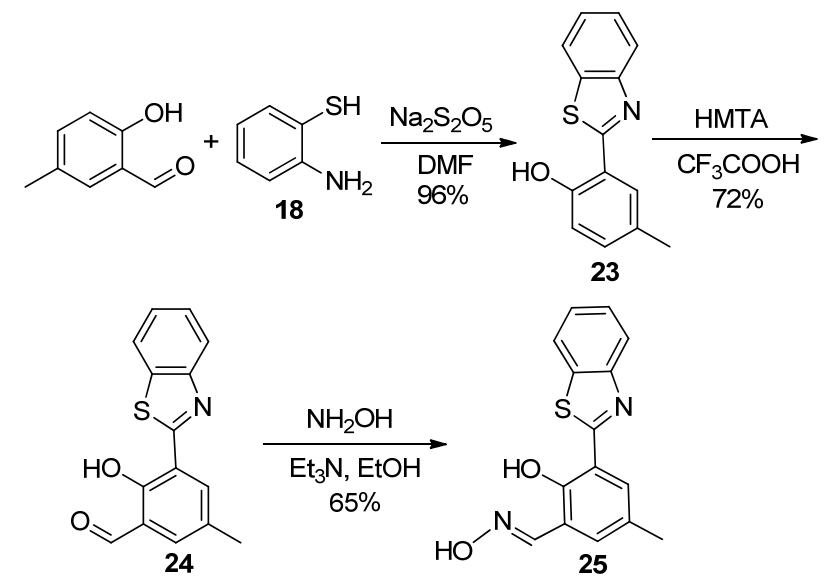

图式 13 探针 25 的合成

Scheme 13 Synthesis of probe 25

郭媛课题组 ${ }^{[31]}$ 则以同样的起始原料合成苯并噻唑 类探针, 但使用环境友好的氧化剂 $\mathrm{H}_{2} \mathrm{O}_{2}$ 在酸性条件下 先生成化合物 23 , 再依次与 HMTA、 $\mathrm{CH}_{3} \mathrm{NO}_{2}$ 反应得到 了探针分子 26 (Scheme 14), 其可用于 $\mathrm{HSO}_{3}^{-}$的识别. 由于从酚羟基到硝基存在显著的 ICT 效应，探针 26 仅 有微弱的苂光. 滴加入 $\mathrm{HSO}_{3}^{-}$后, 由于与硝基相连的 $\mathrm{C}=\mathrm{C}$ 双键和 $\mathrm{HSO}_{3}^{-}$发生亲核加成, ICT 效应减弱, 同时 伴随产生强的 ESIPT 效应, 故荧光发生蓝移, 在 $480 \mathrm{~nm}$ 处有强的荧光, 且肉眼可观察到溶液由浅粉色变无色. 由于探针 26 灵敏性高、抗干扰能力强、斯托克斯位移 大, 被用于细胞内 $\mathrm{HSO}_{3}^{-}$的生物成像.

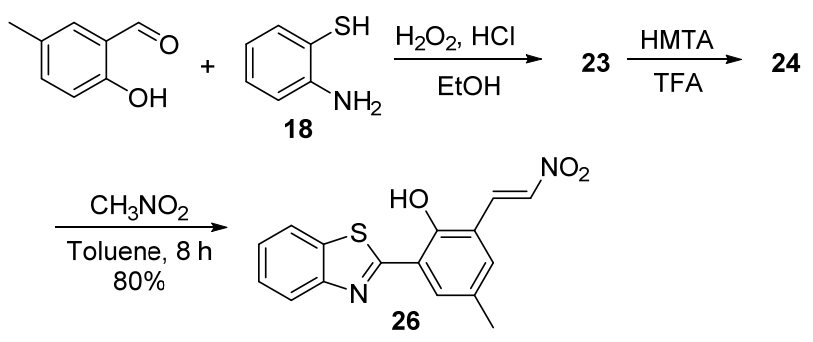

图式 14 探针 26 的合成

Scheme 14 Synthesis of probe 26

张向阳课题组 ${ }^{[32]}$ 则用含醛基的苯并噻唑化合物 24 与肼类反应, 合成了一种能够特异性识别过氧化亚硝酸 离子 $\mathrm{ONOO}^{-}$的腙类荧光探针 27 (Eq. 3). 探针本身呈弱

的荧光，但它的水溶液中若存在 $\mathrm{ONOO}^{-}$, 则形成腙的 $\mathrm{C}=\mathrm{N}$ 双键会被迅速氧化为醛基而转化为化合物 24, ESIPT 效应显著增强, 荧光随之增强. 因此, 探针 27 能 实现对 $\mathrm{ONOO}^{-}$的识别, 响应时间仅需 $60 \mathrm{~s}$, 检测限达 $5.8 \times 10^{-8} \mathrm{~mol} \cdot \mathrm{L}^{-1}$, 并且可用于细胞成像.

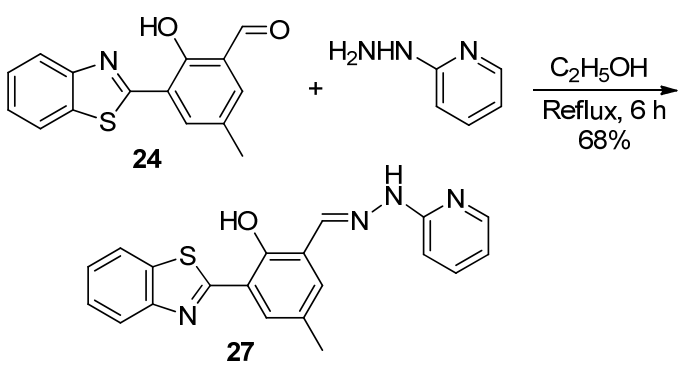

王芳课题组 ${ }^{[33]}$ 将化合物 24 依次与肼、5-甲氧基邻 羟基苯甲醛反应，制备了探针分子 28 (Scheme 15). 探 针 28 本身在 $573 \mathrm{~nm}$ 处有橙色的苂光，在向其溶液中加 入金属离子 $\mathrm{Zn}^{2+}$ 或 $\mathrm{Cd}^{2+}$ 后, 形成的金属配合物使荧光 蓝移至 $520 、 540 \mathrm{~nm}$, 均为黄色. 若向溶液中再加入适量 半胱氨酸, $\mathrm{Cd}^{2+}$ 的金属配合物荧光会发生红移，回复至 原来 $573 \mathrm{~nm}$ 处, 而 $\mathrm{Zn}^{2+}$ 的金属配合物则无此现象, 故 可依此进一步鉴别 $\mathrm{Zn}^{2+} 、 \mathrm{Cd}^{2+}$.

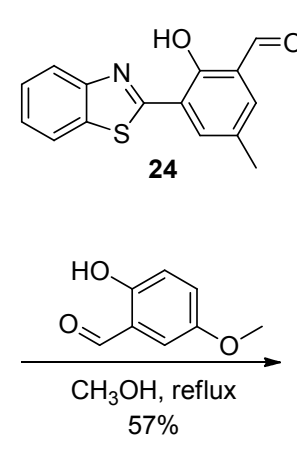

图式 15 探针 28 的合成

Scheme 15 Synthesis of probe $\mathbf{2 8}$

其实, 利用苯并噻唑化合物直接参与反应而构建探 针的报道还很多 ${ }^{[34]}$. 例如, 鲜啟鸣课题组 ${ }^{[35]}$ 用 2-甲基苯 并噻唑类化合物与糠醛衍生物的缩合反应，制备了探针 29 (Scheme 16). 其中, 带正电荷的苯并噻唑部分为吸 电子基团，呋喃基团为供电子基团，共同构成了典型的 $D-\pi-A$ 型分子，具有 ICT 效应. 当分子遇 $\mathrm{CN}^{-}$后，苯并 噻唑中 $\mathrm{C}=\mathrm{N}$ 与 $\mathrm{CN}^{-}$发生亲核加成, 分子内 $\mathrm{ICT}$ 效应被 抑制, 紫外吸收峰发生蓝移, 溶液颜色由黄色变为绿色. 探针 29 对 $\mathrm{CN}^{-}$的检测限为 $1.1 \times 10^{-7} \mathrm{~mol} \cdot \mathrm{L}^{-1}$, 低于世界 卫生组织的 $(\mathrm{WHO})$ 参考浓度 $1.9 \times 10^{-6} \mathrm{~mol} \cdot \mathrm{L}^{-1}$, 故有望 用于真实水样的检测. 
<smiles>CC[n+]1c(C)sc2ccccc21</smiles>

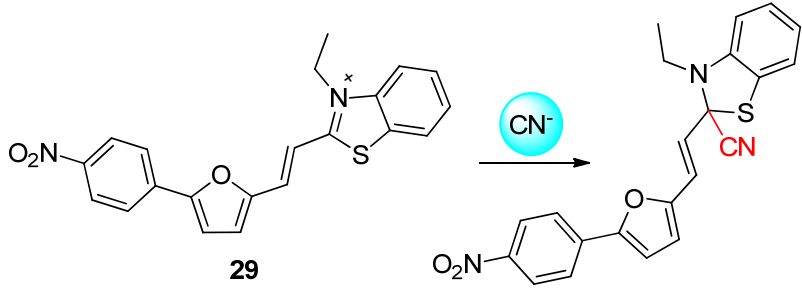

图式 16 探针 29 的合成与作用机理

Scheme 16 Synthesis of probe $\mathbf{2 9}$ and its interaction mechanism

最近, 张劲等 ${ }^{[36]}$ 以 2-(2-羟基苯基)苯并噻唑为原料, 常温下一步反应合成了可用于活体细胞中 $\mathrm{HPO}_{4}{ }^{2-}$ 检测 的草酸酯型探针 30 (Scheme 17). 该探针能在二甲基亚 砜(DMSO)/4-差弪乙基哌嗪乙磺酸(HEPES)的缓冲溶液中 与 $\mathrm{HPO}_{4}^{2-}$ 发生特异性水解, 生成强荧光化合物, 溶液 由无色透明变为亮蓝色, 并伴随有 $76 \mathrm{~nm}$ 的紫外吸收峰 红移, 苂光增强达 210 倍, 但对其他阴离子 $\mathrm{F}^{-} 、 \mathrm{ClO}_{4}^{-}$、 $\mathrm{S}_{2} \mathrm{O}_{3}^{2-}$ 均无明显响应. 含有苯并噻唑结构的化合物也能 与二硼酸酯化合物偶联聚合, 得到共轭聚合物型的荧光 探针，如一种荧光比率型探针 31. 基于分子的 ICT 效应, 探针 31 在乙醇溶液中能特异性识别 $\mathrm{Zn}^{2+}$, 检测限达 $1.0 \times 10^{-8} \mathrm{~mol} \cdot \mathrm{L}^{-1[37]}$.
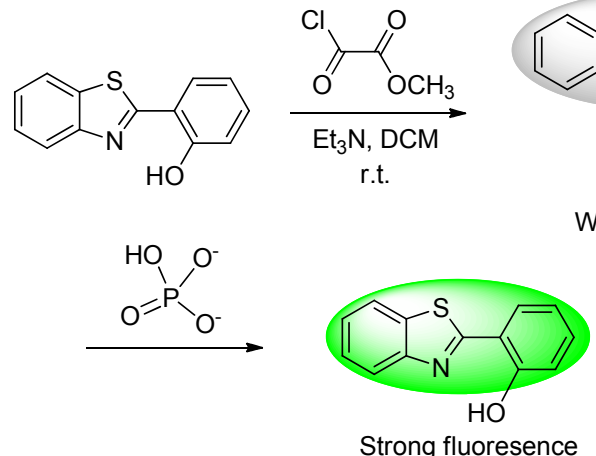

图式 17 探针 $\mathbf{3 0}$ 的合成与作用机理

Scheme 17 Synthesis of probe $\mathbf{3 0}$ and its interaction mechanism

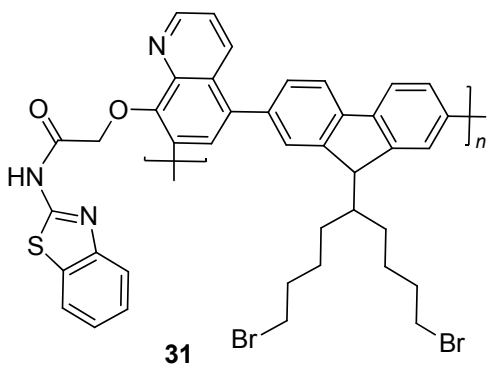

当然，芳香醛类化合物也是合成各种苯并噻唑基苂
光探针的常见起始原料 ${ }^{[38]}$, 若是使用稠环芳香醛更有 利于增强苂光效果. 例如, Yoon 等 ${ }^{[39]}$ 用 2-羟基萠甲醛与 邻氨基苯硫酚反应, $\mathrm{H}_{2} \mathrm{O}_{2}$ 作氧化剂，制备了一种用于检 测硝基芳香族化合物(NACs)的探针 32. 该探针在高极 性的溶液(DMSO、DMF 等)中，由于充分的去质子化， 而有强烈的荧光. 当其与 NACs 类化合物接触时, 发生 质子化作用，探针会形成原来的分子形式，苂光发生猝 灭，故能实现对 NACs 的 on-off 型传感.

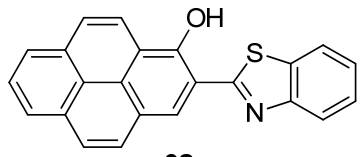

32

\section{3 苯并咪唑类荧光探针}

同为苯并五元氮杂环的苯并咪唑，在荧光检测领域 也有着更为广泛的应用 ${ }^{[40]}$ 。它们的合成方法与苯并噁 唑、苯并噻唑的合成颇为相似 ${ }^{[41]}$, 较常见的是通过邻苯 二胺与羧酸缩合反应制备 ${ }^{[42]}$. 其中, 利用芳香二羧酸可 以开发出含有 2 个苯并咪唑结构的金属探针 ${ }^{[43]}$. 本课题 组开发了能在半水介质中对 $\mathrm{Ag}^{+} 、 \mathrm{Fe}^{3+}$ 离子实现双功能 识别探针分子 $\mathbf{3 3}^{[44]}$.

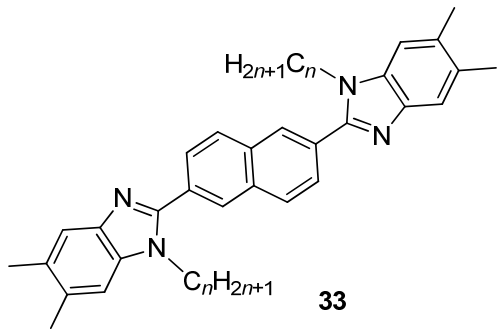

各类醛与邻苯二胺反应也是生成苯并咪唑的常用 方法 ${ }^{[45]}$. 例如谢志刚等 ${ }^{[46]}$ 用含吡咯基团的芳香醛与邻 苯二胺 34 反应, 在对甲苯磺酸催化下, 得到一种 $\mathrm{pH}$ 传 感的探针 35 (Eq. 4). 由于苯并咪唑基团的供电子效应, 化合物 35 的苂光和紫外吸收有明显的红移. 当与酸作 用发生质子化后苂光增强, 并且和紫外吸收产生大的蓝 移现象, 溶液由黄色变为绿色. 因此, 根据探针 $\mathbf{3 5}$ 苂光 的 off-on 型变化, 能实现对 $\mathrm{pH}$ 的监测.

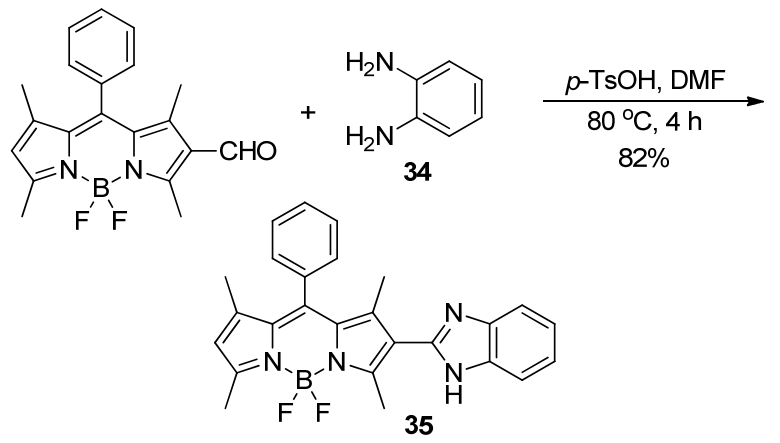


具有大共轭结构的稠环芳香醛，也常用于制备苯并 咪唑型探针 ${ }^{[47]}$. 例如马立军课题组 ${ }^{[48]}$ 用萠甲醛与 34 在 室温下反应, 制备了探针 36 (Eq. 5). 探针 $\mathbf{3 6}$ 能高选择 性地识别 $\mathrm{Fe}^{3+}$, 在向其水溶液中逐滴加入 $\mathrm{Fe}^{3+}$ 至 30 equiv. 时, 探针 36 与 $\mathrm{Fe}^{3+}$ 以 $2: 1$ 络合, 荧光呈现 turn on 型变化, 增强高达 510 倍, 检测限为 $2.0 \times 10^{-7} \mathrm{~mol} \cdot \mathrm{L}^{-1}$.

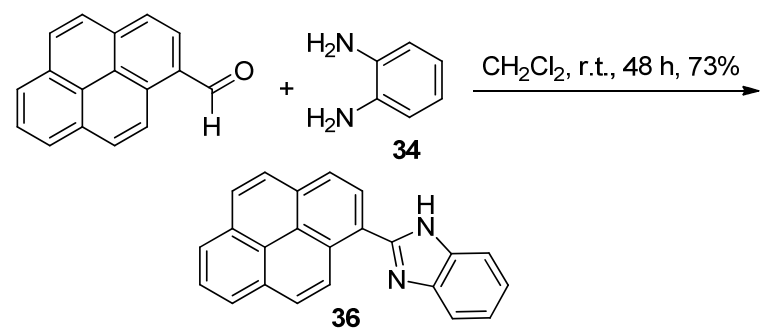

多个醛基的化合物参与构建苯并咪唑型的苂光探 针, 也有较多的报道 ${ }^{[49]}$. 郑立炎课题组 ${ }^{[50]}$ 用含四个醛基 的芳香化合物与 34 反应, 制备了分子内同时含四个苯 并咪唑基的探针 37 (Eq. 6). 探针 37 有聚集诱导发光 (AIE)效应，对能与苯并咪唑有强鳌合作用并形成团聚 体的 $\mathrm{Ag}^{+}$有特异性识别. 在向溶液中滴入 $\mathrm{Ag}^{+}$, 探针分 子 37 能与 $\mathrm{Ag}^{+}$发生 $1: 2$ 络合, 并伴随产生强的黄色荧 光信号, 对 $\mathrm{Ag}^{+}$的检测限为 $6.0 \times 10^{-6} \mathrm{~mol} \cdot \mathrm{L}^{-1}$.<smiles>O=Cc1ccc(C(=C(c2ccc(C=O)cc2)c2ccc(C=O)cc2)c2ccc(C=O)cc2)cc1</smiles>

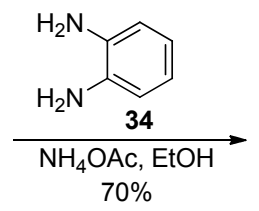<smiles>c1ccc(C(=C(c2ccc(-c3nc4ccccc4[nH]3)cc2)c2ccc(-c3nc4ccccc4[nH]3)cc2)c2ccc(-c3nc4ccccc4[nH]3)cc2)cc1</smiles>

当然, 对于苯并咪唑类荧光探针的研究, 直接使用 苯并咪唑类化合物与羧酸或其衍生物 ${ }^{\left[{ }^{[} 1,52\right]}$ 、醛 ${ }^{[33]} 反$ 应构 建性能各异的探针分子的报道也比较常见. 例如, Goswami 课题组 ${ }^{[54]}$ 直接用两分子 2-氯甲基-1-甲基苯并咪唑 与罗丹明酰肼反应, 合成了一种可选择性检测 $\mathrm{Cu}^{2+}$ 的 探针 38 (Eq. 7). 与 $\mathrm{Cu}^{2+}$ 络合后, 分子内发生从苯并咪唑 到罗丹明的苂光共振能量转移效应(FRET), 表现出明 显的吸收和发射变化, 从而有效地检测 $\mathrm{Cu}^{2+}$, 检测限为 $3.1 \times 10^{-6} \mathrm{~mol} \cdot \mathrm{L}^{-1}$.

在衍生化构建苯并咪唑类探针的报道中, 含有氨基

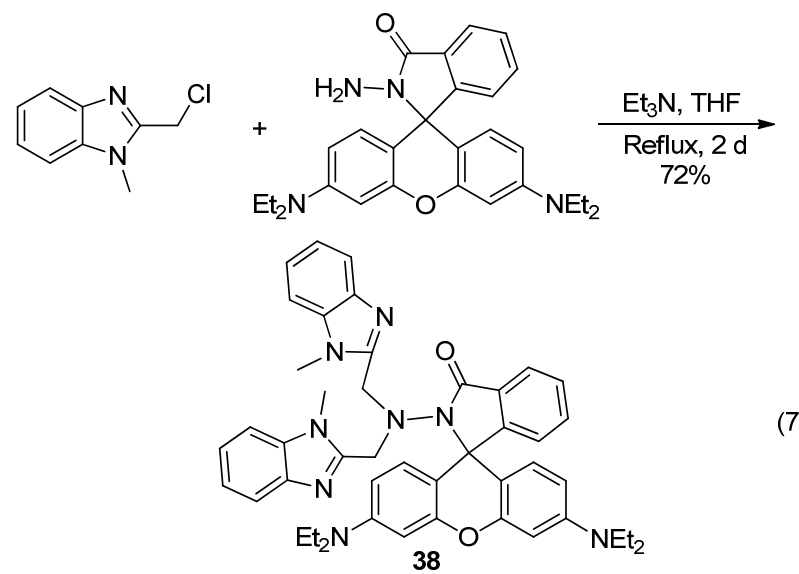

(7)

的苯并咪唑化合物最为常见. 例如, 高春梅课题组 ${ }^{[55]}$ 用 2-氨甲基苯并咪唑与 2-喹啉甲酰氯回流反应 $2 \mathrm{~h}$, 合成 了可特异性识别 $\mathrm{Ag}^{+}$的探针分子 39 (Eq. 8); 而 Das 等 ${ }^{[56]}$ 则用 2-氨基苯并咪唑与 2-羟基-1-荎甲醛回流反应 $6 \mathrm{~h}$, 制备了检测 $\mathrm{Zn}^{2+} 、 \mathrm{~N}^{3-}$ 的探针分子 $\mathbf{4 0}$ (Eq. 9).<smiles>NCc1nc2ccccc2[nH]1</smiles><smiles>O=C(Cl)c1ccc2ccccc2n1</smiles>

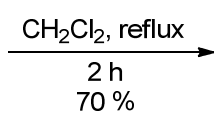<smiles>O=C(NCc1nc2ccccc2[nH]1)c1ccc2ccccc2n1</smiles><smiles>Nc1nc2ccccc2[nH]1</smiles><smiles>Oc1c(/C=N/c2nc3ccccc3[nH]2)ccc2ccccc12</smiles>

类似地，利用苯并咪唑化合物 $\mathbf{4 1}$ 中氨基的反应，可 合成系列检测双离子的苯并咪唑类探针 (Scheme 18). 例如, 探针 42 可细胞内检测 $\mathrm{Cu}^{2+} 、 \mathrm{~S}^{2-[57]}$, 探针 43 用于 检测 $\mathrm{Al}^{3+} 、 \mathrm{Cd}^{2+[58]}$, 探针 44 同时检测 $\mathrm{Hg}^{2+} 、 \mathrm{Cu}^{2+[59]}$.

\section{4 苯并吡咯类荧光探针}

苯并吡咯又称吲哚，不同于苯并惡唑、苯并噻唑、 苯并咪唑, 吲哚分子内只含有一个 $\mathrm{N}$ 杂原子. 由于吡咯 环上能吸收特征频率光的 $\mathrm{C}=\mathrm{C}$ 发色团存在, 吲哚类化 合物同样具有良好的苂光性能 ${ }^{[60]}$. 直接利用吲哚本 身 $^{[61]}$, 或芳环上不同位置上的羧基、醛基等 ${ }^{[62,63]}$ ，与其 它化合物发生加成、缩合反应等，也能构建不同性能的 荧光探针.

Sakhuja 课题组 ${ }^{[64]}$ 用 3-吲哚乙酸与含有氨基的香豆 素衍生物发生酰胺化反应, 制备了能选择性识别 $\mathrm{Hg}^{2+}$ 

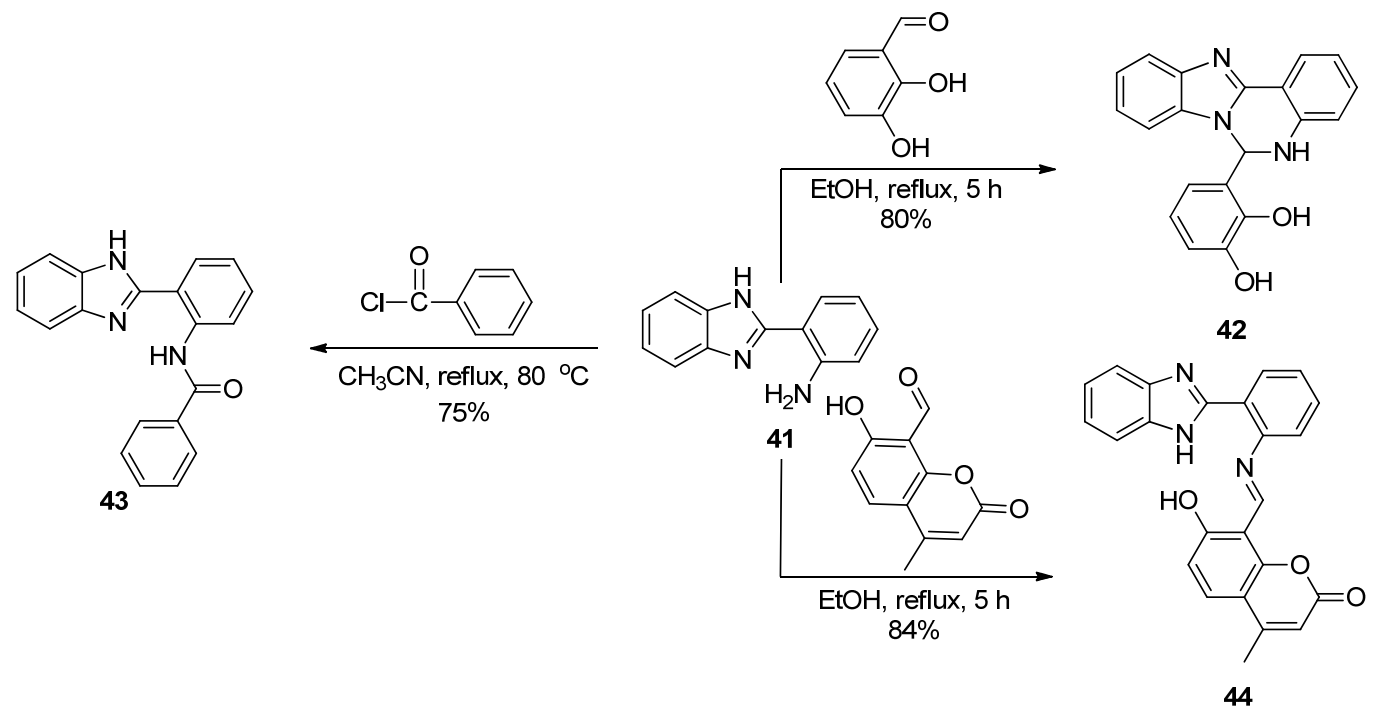

图式 18 探针 $42 \sim 44$ 的合成

Scheme 18 Synthesis of probes $42 \sim 44$

的探针分子 45 (Eq. 10); Chen 等 ${ }^{[65]}$ 利用含有醛基的吲哚 化合物与丙二腈发生缩合反应, 制备出 $\mathrm{CN}^{-}$检测的比色 及比率型探针分子 46 (Eq. 11).<smiles>O=C(O)Cc1c[nH]c2ccccc12</smiles>

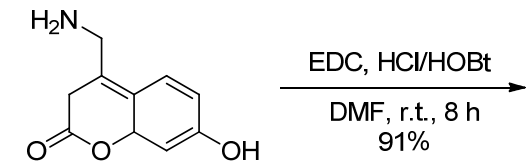<smiles>CS(=O)C1CC(CNC(=O)Cc2c[nH]c3ccccc23)=C2C=CC(O)=CC2O1</smiles><smiles>Cn1ccc2cc(C=C(C#N)C#N)ccc21</smiles>

实际上, 利用 3-吲哚甲醛类化合物的各种反应, 设 计含有 $\mathrm{C}=\mathrm{C}^{[66]} 、 \mathrm{C}=\mathrm{N}^{[67]}$ 双键的探针, 更为常见. 例如, 土耳其 Cosut 课题组 ${ }^{[68]}$ 用 2-甲基-3-吲哚甲醛与氟硼二吡 咯类化合物发生缩合反应，合成了苂光探针分子 47 (Eq. 12). 借助氟嗍二吡咯类化合物荧光光谱峰窄、光学
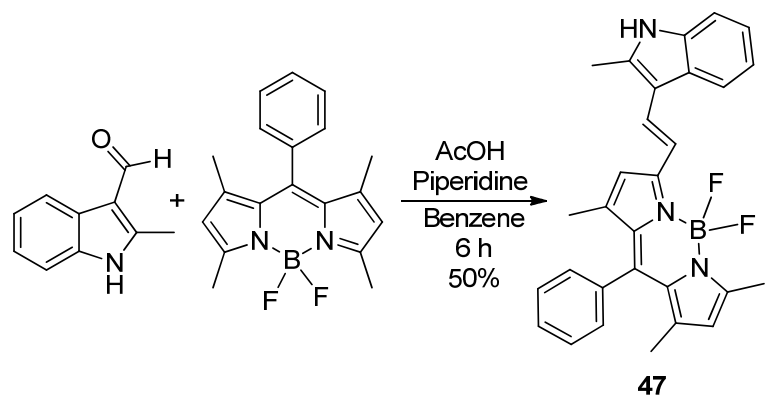

性质稳定的特点, 探针 47 可实现苂光 on-off 型变化检测 $\mathrm{Cu}^{2+}$.

利用 3-吲哚甲醛类化合物与二胺的缩合反应，可构 建含有多个 $\mathrm{C}=\mathrm{N}$ 双键的探针分子 ${ }^{[69]}$. Chellappa 课题 组 ${ }^{[70]}$ 将 3-吲哚甲醛与 2,3-二氨基-2-丁烯二腈缩合, 制备 了 $\mathrm{F}^{-}$探针 48 (Eq. 13), 其与 $\mathrm{F}^{-}$的配位比为 $1: 1$, 检测限 为 $2.73 \times 10^{-7} \mathrm{~mol} \cdot \mathrm{L}^{-1}$.

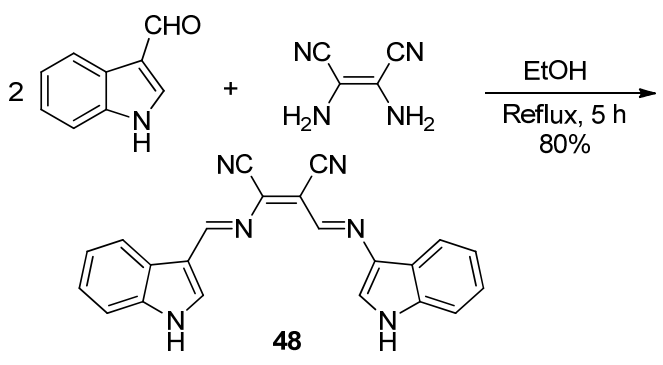

\section{5 咔唑类荧光探针}

咔唑是一类富电子的芳香性氮杂环化合物，其分子 内的电子转移较强, 并且共轭体系较大, 具有良好的光 稳定性. 近年来，咔唑及其衍生物分子因其独特的刚性 稠杂环结构和活跃的分子内电荷转移, 已被广泛应用于 苂光探针分子的设计、合成 ${ }^{[71 \sim 74]}$.

Kim 课题组 ${ }^{[75]}$ 以 3-碘-9-甲基咔唑为原料, 经两步 反应, 在咔唑的 3,6 号位分别引入醛基和羧基. 在此基 础上再分两步构建了含苯并噻唑基的咔唑类探针 49 . 在水溶液体系中探针 49 能实现对 $\mathrm{MnO}_{4}^{-}$的荧光 turn on 型检测, 菼光量子产率从 0.011 增加至 0.32 , 检测限可 达 $3.2 \times 10^{-9} \mathrm{~mol} \cdot \mathrm{L}^{-1}$. 其机理是探针分子在氧化作用下 生成了强荧光性的醌式物质 50 (Scheme 19). 


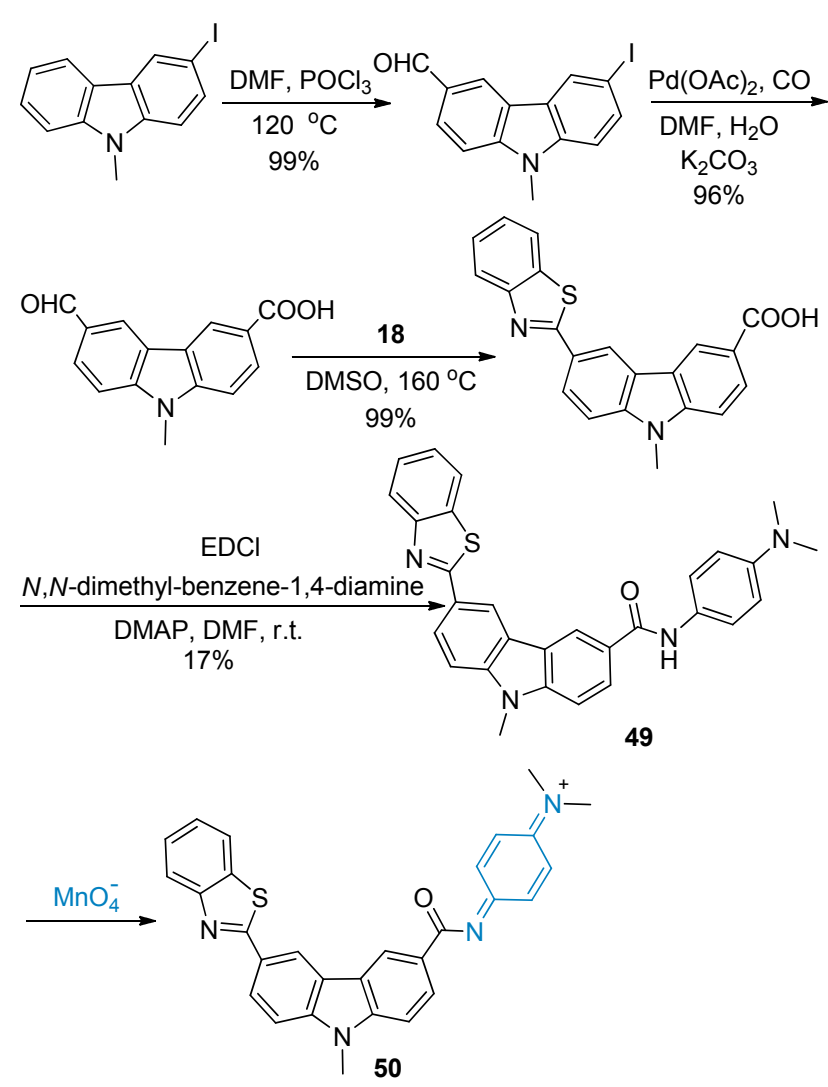

图式 19 探针 49 的合成与作用机理

Scheme 19 Synthesis of probes 49 and its interaction mechanism

除利用咔唑 3-(或 6-)位的醛基直接构建同时含有苯 并噻唑与咔唑官能团的菼光探针外 ${ }^{[76]}$ ，也可利用 2-氰甲 基苯并噻唑的活泼亚甲基与咔唑 3-位醛基缩, 合构建这 类同时含有 2 种稠合氮杂环的苂光探针 ${ }^{[77]}$. 利用类似的

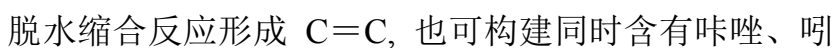
哚类的荧光探针 ${ }^{[78]}$. 当然, 也可利用醛基与氨基的脱水 缩合反应，构建含有 $\mathrm{C}=\mathrm{N}$ 的咔唑类荧光探针 ${ }^{[79]}$.

另外，用含氨基的咔唑化合物与各类物质反应，设 计得到功能性的探针分子也常有报道 ${ }^{[80]}$. 蒋长龙课题 组 ${ }^{[81]}$ 用 3-氨基-9-乙基咔唑 51 与两分子溴代乙酸乙酯发 生反应，先合成了得到双取代的中间化合物，再将中间 体进一步与肼反应, 得到一种可连续检测 $\mathrm{Cu}^{2+}$ 和 $\mathrm{S}^{2-}$ 的 探针 52 (Scheme 20); 胡胜利等 ${ }^{[82]}$ 则同样利用 51 中的咔 唑基苂光团, 以邻苯二甲酰亚胺为识别和结合位点, 设 计合成了探针分子 53 , 其能通过盖布瑞尔反应机理识 别肼，同时释放出化合物 51 (Scheme 21).

\section{2 苯并六元氮杂环型荧光探针}

苯并六元氮杂环主要是苯并吡啶(喹啉)、苯并吡嗪、 吩嗪等芳香化合物. 喹啉、吡嗪、吩嗪也是一类性能优 良的发光基团, 在荧光探针分子的设计与合成中同样颇
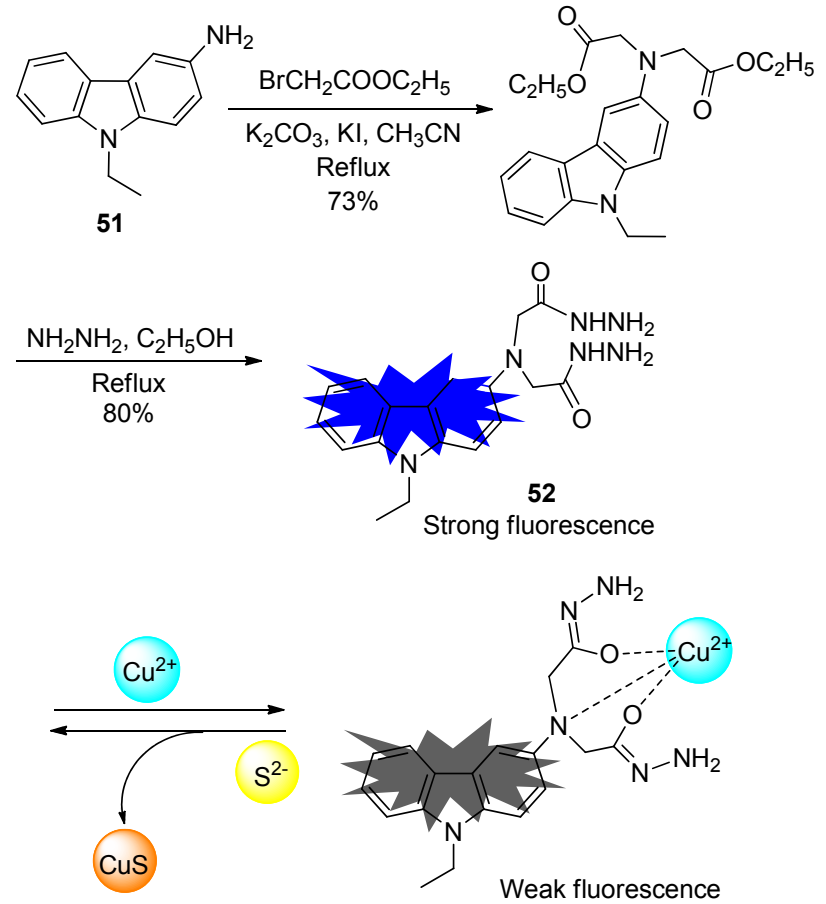

图式 20 探针 $\mathbf{5 2}$ 的合成与作用机理

Scheme 20 Synthesis of probe $\mathbf{5 2}$ and its interaction mechanism
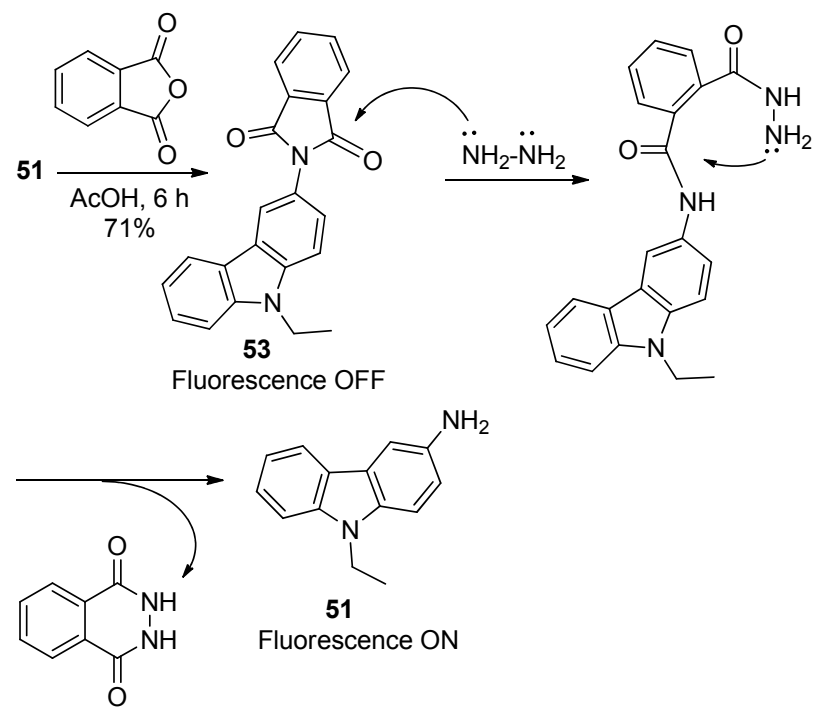

图式 21 探针 53 的合成与作用机理

Scheme 21 Synthesis of probes $\mathbf{5 3}$ and its interaction mechanism

为常见 ${ }^{[83]}$, 用于不同分析物的传感.

\section{1 苯并吡啶类荧光探针}

苯并吡啶包括喹啉、异喹啉两类，其中，喹啉类化 合物更多的被报道于苂光传感领域 ${ }^{[84]}$ ，且利用喹啉上 不同位置的取代基(如羟基 ${ }^{[85]}$ 、氨基等)进行反应，可获 得性能良好、基于喹啉骨架的苂光探针分子. 特别是 8着基喹啉类化合物，由于其良好的光稳定性、配位能力， 
在荧光探针的设计合成中常被广泛使用 ${ }^{[86,87]}$.

徐括喜课题组 ${ }^{[88]}$ 用 2 -差弪甲基- 8 -差欮基-喹啉与具有较 强结合能力的吡啶衍生物发生取代反应, 制备了一种 turn on 型检测 $\mathrm{Cd}^{2+}$ 的探针 54 (Eq. 14). 得益于良好的水 溶性, 探针 54 能在几乎纯水溶液中实现对 $\mathrm{Cd}^{2+}$ 的传感, 检测限达 $1.2 \times 10^{-6} \mathrm{~mol} \cdot \mathrm{L}^{-1}$. 在对活细胞的研究中, 探 针 54 几乎没有毒性, 能正常地通过细胞膜, 用来检测活 细胞中 $\mathrm{Cd}^{2+}$.

类似地，8-氨基喹啉由于良好的光谱特性，也常用 于探针分子的构建 ${ }^{[89]}$. Wong 课题组 ${ }^{[90]}$ 则用 8 -氨基喹啉 与氯代乙酰氯、二异丙基胺依次进行取代反应，合成了 探针 55. 在 55 的溶液中滴加 $\mathrm{Zn}^{2+}$, 苂光显著增强. 这是 因为探针 55 与 $\mathrm{Zn}^{2+}$ 作用后, 原本使猝灭苂光的 PET 效 应被抑制, 导致荧光增强(Scheme 22). 探针 55 对 $\mathrm{Zn}^{2+}$ 检测限为 $4.1 \times 10^{-8} \mathrm{~mol} \cdot \mathrm{L}^{-1}$.

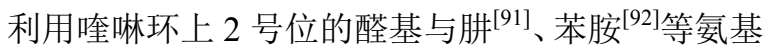
化合物反应, 是构建席夫碱类苂光探针的有效手段之 一. 例如, Ramu 等 ${ }^{[93]}$ 用对甲基苯酰肼的反应, 合成了探 针分子 56 (Eq. 15). 在 $\mathbf{5 6}$ 的 DMSO 溶液中加入 $\mathrm{Mg}^{2+}$, 形成的金属配合物内部存在 ICT 效应，溶液在 $474 \mathrm{~nm}$
的苂光发生猝灭，但在 $590 \mathrm{~nm}$ 出现新的发射峰，且其荧 光强度不受其他金属离子的影响. 因此，探针 56 能在其 他碱土金属离子存在的情况下，高灵敏地检测 $\mathrm{Mg}^{2+}$, 检测限可达 $4.7 \times 10^{-8} \mathrm{~mol} \cdot \mathrm{L}^{-1}$.

将喹啉与以长波发射、高量子产率著称的罗丹明苂 光团结合，也是设计合成新型菼光探针的巧妙方法 ${ }^{[94]}$. Adhikari 等 ${ }^{[95]}$ 通过长度控制配位选择性的特点, 设计、 合成了基于 FRET 机理的 $\mathrm{Bi}^{3+}$ 苂光探针 57 (Eq. 16).

在喹啉环基础上继续构建苯并五元氮杂环，可设 计、合成出能同时识别 $\mathrm{Cu}^{2+} 、 \mathrm{Cd}^{2+}$ 的双功能探针分子

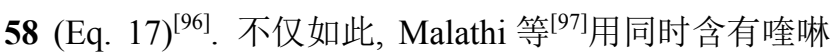
与苯并咪唑结构的原料与 2-颈基乙醇发生亲核取代反 应，合成了能用于 $\mathrm{pH}$ 传感、金属离子检测、生物成像 等的多功能苂光探针分子 $\mathbf{5 9}$ (Eq. 18).

芳环上含两个卤原子的喹啉衍生物, 还能应用于构 建聚合物型苂光探针分子. 冯丽恒课题组 ${ }^{[98]}$ 用 5,7-二 溴-8-甲氧基喹啉与苯二嗍酸酯发生偶联聚合反应，得 到了选择性识别 $\mathrm{Fe}^{3+}$ 的探针 60 (Eq. 19). 聚合物探针分 子 60 具有较高的苂光量子产率 $(0.74)$, 与 $\mathrm{Fe}^{3+}$ 发生配位

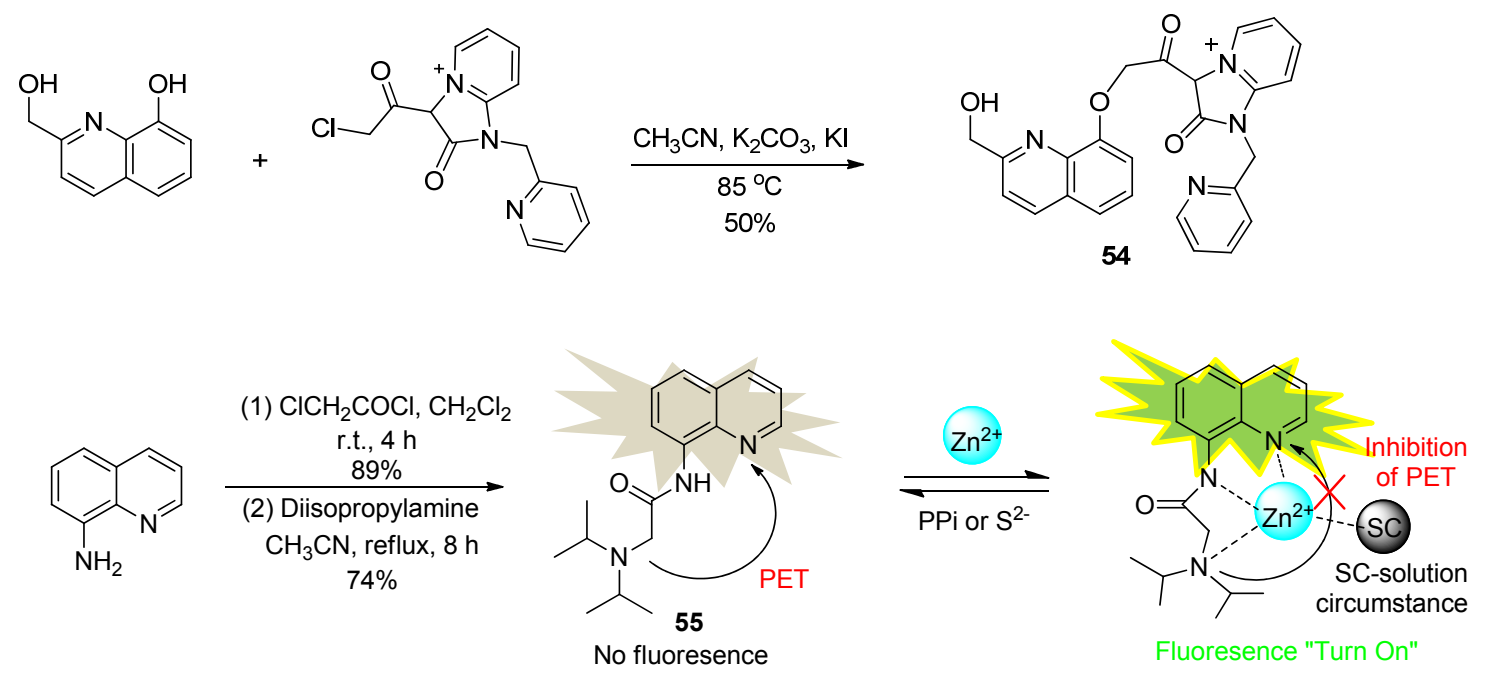

图式 22 探针 $\mathbf{5 5}$ 的合成和作用机理

Scheme 22 Synthesis of probe $\mathbf{5 5}$ and its interaction mechanism<smiles>Cc1ccc(C(=O)NN)cc1</smiles>

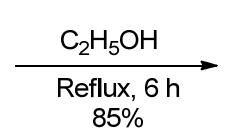<smiles>Cc1ccc(C(=O)N/N=C\c2ccc3cccc(O)c3n2)cc1</smiles>

56 
<smiles>CCOC(=O)COc1cccc2ccc(C=O)nc12</smiles><smiles>[R16][R16]([H])([H])C(CO)CO</smiles><smiles></smiles><smiles>Clc1nc2ccccc2cc1-c1nc2ccccc2[nH]1</smiles><smiles>OCCS</smiles>

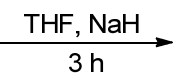

59
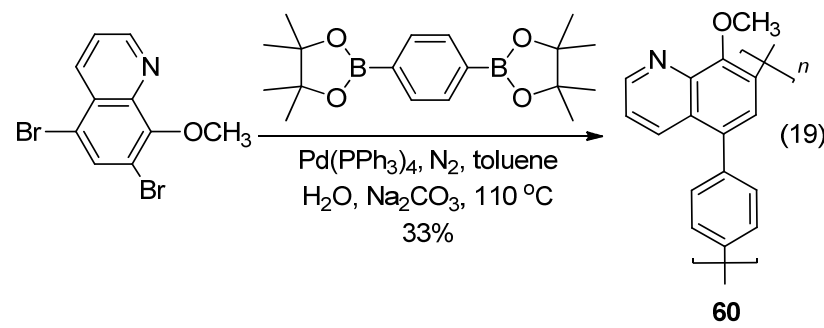

后, 由于分子内部的 PET 效应, 其苂光逐渐发生猝灭, 对 $\mathrm{Fe}^{3+}$ 的检测限为 $1.0 \times 10^{-8} \mathrm{~mol} \cdot \mathrm{L}^{-1}$.

\section{2 苯并吡嗪类荧光探针}

苯并吡嗪又称喹喔啉, 不同于喹啉的是, 其苯环一 侧的吡嗪有两个 $\mathrm{N}$ 原子, 故具有更强的配位能力. 目前,

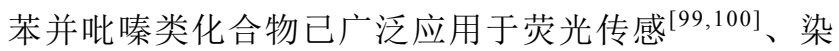
料 ${ }^{[101]}$ 、材料等领域. 苯并吡嗪类苂光探针常见的合成方

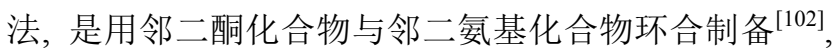
如 Bhosale 课题组 ${ }^{[103]}$ 对 $\mathrm{pH}$ 检测探针 61 的合成(Eq. 20).<smiles>N#CC(C#N)=C(c1ccccc1)c1ccc2nc(-c3ccc(Br)cc3)c(-c3ccc(Br)cc3)nc2c1</smiles>

构建同时含有苯并吡嗪和其他苯并氮杂环结构的 苂光探针，可应用于各种离子的检测 ${ }^{[104]}$. 例如，韩辉课 题组 ${ }^{[105]}$ 用含喹啉苂光团的邻二酮类与邻苯二胺反应, 制备了一系列能用于 $\mathrm{Zn}^{2+}$ 检测的苂光 turn on 型探针分 子, 如探针 62 (Eq. 21).
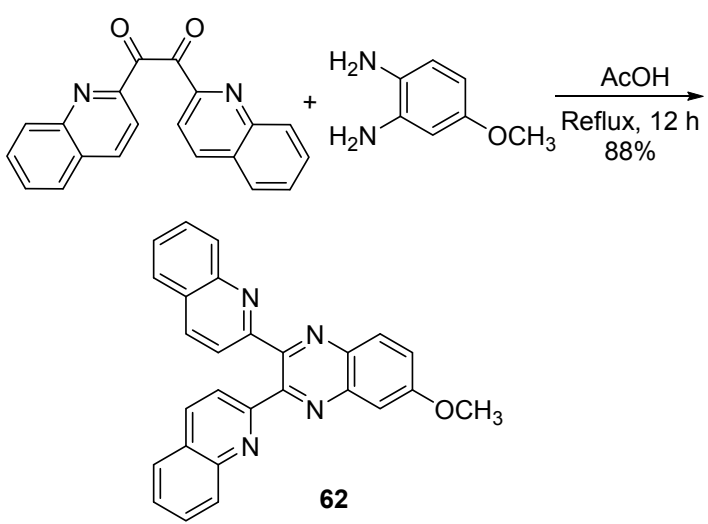

基于苯并吡嗪类化合物构建探针分子的研究，近来 较多 ${ }^{[106,107]}$. 例如, 覃兆海课题组 ${ }^{[108]}$ 用含溴原子的苯并 吡嗪化合物与 2-甲硫基苯嗍酸发生 Suzuki 交叉偶联反 应，合成了配位型检测 $\mathrm{Pd}^{2+}$ 的探针分子 63 (Eq. 22). 在 纯水溶液中, 探针 63 与作为软金属离子的 $\mathrm{Pd}^{2+}$ 具有强 的配位作用, 致使溶液的苂光发生猝灭. 但在溶液中继 续加入 $\mathrm{CN}^{-}$后, 溶液的绿色苂光又得以恢复, 这是由于 $\mathrm{Pd}^{2+}$ 与 $\mathrm{CN}^{-}$形成了更稳定的络合物. 在实际水样中, 探 针 63 对 $\mathrm{Pd}^{2+}$ 的检测限可达 $2.2 \times 10^{-8} \mathrm{~mol} \cdot \mathrm{L}^{-1}$.

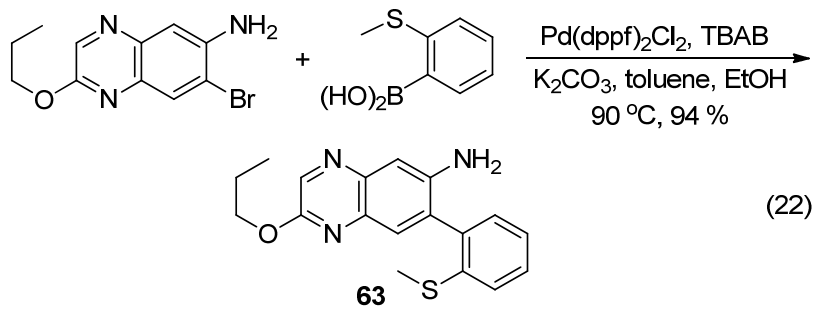

另外，基于卤代苯并吡嗪化合物的金属催化偶联反 应设计聚合型苂光探针，也有报道 ${ }^{[109]}$. 曹德榕课题组 [110]则用含吡啶基团的苯并吡嗪衍生物与含炔基的咔唑 化合物通过 Sonogashira 反应，制备了共轭聚合物型探 针分子 64 (Eq. 23), 其能可视化识别 $\mathrm{Ag}^{+}$, 对 $\mathrm{Ag}^{+}$的检 测限为 $5.0 \times 10^{-7} \mathrm{~mol} \cdot \mathrm{L}^{-1}$.

\section{3 吩嗪类荧光探针}

吩嗪又称为二苯并吡嗪. 有别于苯并吡嗪, 其吡嗪 环两侧存在两个苯环，共轭程度更大，是良好的荧光发 色团，可设计、合成荧光探针分子，用于金属离

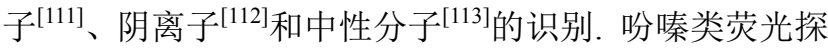
针的合成方法, 最常见的是以邻苯二胺 34 为原料, 先制 
<smiles>Brc1ccc(Br)c2nc(-c3ccccn3)c(-c3ccccn3)nc12</smiles>

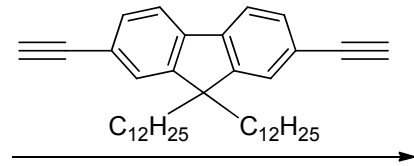

$\mathrm{Pd}\left(\mathrm{PPh}_{3}\right)_{4}$, Cul

$60{ }^{\circ} \mathrm{C}, 36 \mathrm{~h}$ $60 \%$

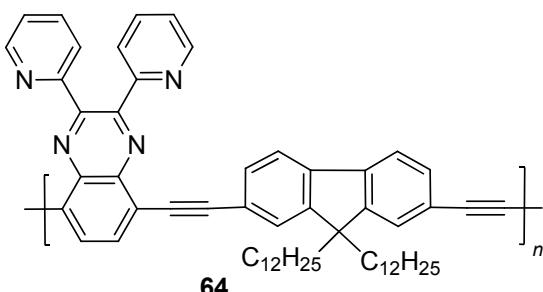

备 2,3-二氨基吩嗪 $\mathbf{6 5}^{[114]}$, 再与芳香醛缩合 ${ }^{[115]}$, 如西北 师范大学魏太保课题组 ${ }^{[116]}$ 制备的能连续检测 $\mathrm{Fe}^{3+}$ 、 $\mathrm{H}_{2} \mathrm{PO}_{4}^{-}$的探针 66 (Scheme 23).
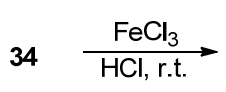<smiles>Nc1cc2nc3ccccc3nc2cc1N</smiles>

65
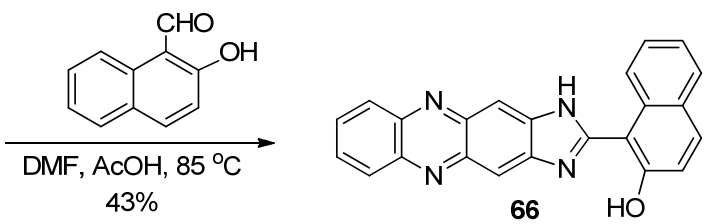

图式 23 探针 66 的合成

Scheme 23 Synthesis of probe $\mathbf{6 6}$

另外, 邻苯二胺 34 还可与 2-羟基-1,4-萘醌反应, 得 到中间体苯并吩嗪化合物, 再与狮三酮在冰醋酸条件下 缩合, 亦可得到苯并吩嗪类探针分子 ${ }^{[117]}$. Khurana 课题 组 ${ }^{[118]}$ 制备的探针 67 (Scheme 24), 在 $0.5 \mathrm{~mol} \cdot \mathrm{L}^{-1}$ 的 $\mathrm{H}_{2} \mathrm{SO}_{4}$ 溶液中苂光量子产率为 0.546 . 当向探针 67 的溶 液中加入 1 equiv. 的 $\mathrm{Cu}^{2+}$ 或 $\mathrm{Pb}^{2+}$ 后, 探针 67 的荧光发生 不同程度的猝灭. 若在溶液中继续滴入乙二胺四乙酸, 探针分子 67 的苂光又得以恢复.

\section{3 结束语}

总之, 随着近年来苂光探针的迅猛发展, 基于苯并 氮杂环设计、合成的探针已有大量成功的实例, 并广泛 应用于各种小分子 $\left(\mathrm{NACs} 、 \mathrm{~N}_{2} \mathrm{H}_{4} \text { 等 }\right)^{[39,82]} 、 \mathrm{pH}^{[19,46]}$ 以及 溶液中同时含多种离子 $\left(\mathrm{Hg}^{2+}-\mathrm{Cu}^{2+[59]} 、 \mathrm{Cu}^{2+}-\mathrm{Cd}^{2+[96]}\right.$ 等 $)$ 的分析物检测, 在生物体内 ${ }^{[27,31]}$ 与纯水相介质 ${ }^{[21,108]}$ 的 检测应用亦有报道. 由于其特有的大共轭结构, 含 $\mathrm{N}$ 、 $\mathrm{O} 、 \mathrm{~S}$ 等杂原子结合位点的存在, 以及其芳环上特定位 置 ${ }^{[68,75]}$ 可设计发生一系列化学反应而引入功能性基团. 特别是通过各种连接臂有效将苯并五元 ${ }^{[55]}$ 、六元 ${ }^{[58]}$ 或多 元 $^{[88]}$ 含氮杂环同时引入分子中, 往往能得到功能多样
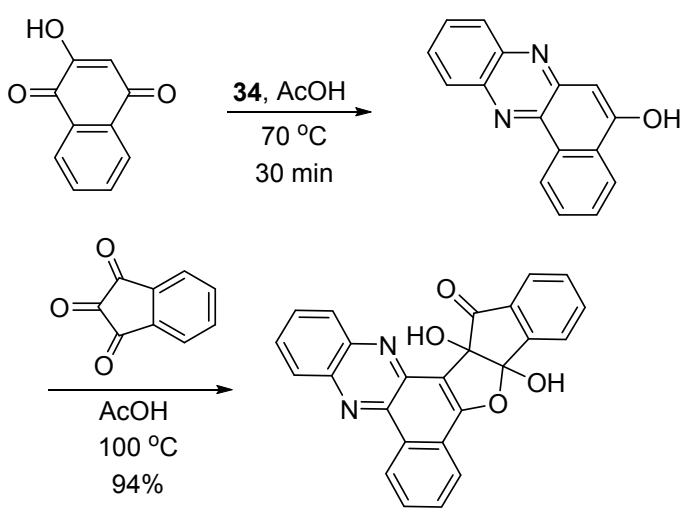

67

图式 24 探针 67 的合成

Scheme 24 Synthesis of probes 67

的探针分子. 在设计、合成方面，苯并氮杂环化合物探 针所涉及的原料、反应类型也越来越丰富多样，反应趋 向于原子经济性与绿色化, 合成路线更加要求设计巧 妙、简洁高效.

展望未来, 如何将具有不同结构、不同功能的基团 组合搭配到一起，构建更为丰富的探针库，特别是同时 含有多个配位点和具有不同响应机理(如 $\mathrm{PET}^{[11,119]}$ 、

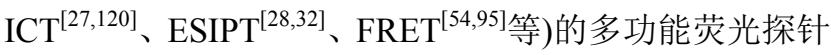
的合成研究，仍须继续得到足够的重视.

\section{References}

[1] Malapati, P.; Krishna, V. S.; Nallangi, R.; Srilakshmi, R. R.; Sriram, D. Eur. J. Med. Chem. 2018, 145, 23.

[2] Wei, P.-F.; Qi, M.-Z.; Wang, Z.-P.; Ding, S.-Y.; Yu, W.; Liu, Q.; Wang, L.-K.; Wang, H.-Z.; An, W.-K.; Wang, W. J. Am. Chem. Soc. 2018, 140,4623

[3] Garreffi, B. P.; Guo, M. L.; Tokranova, N.; Cady, N. C.; Castracane, J.; Levitsky, I. A. Sens. Actuators, B 2018, 276, 466.

[4] Karman, M.; Verde-Sesto, E.; Weder, C. ACS Macro Lett. 2018, 7, 1028.

[5] Bing, Q. J.; Wang, L.; Li, D. L.; Wang, G. Spectrochim. Acta, Part A 2018, 202, 305.

[6] Liu, K.; Guo, P.; Liu, L. J.; Shi, X. F. Sens. Actuators, B 2018, 250 , 667.

[7] Rostovtseva, I. A.; Chernyshev, A. V.; Tkachev, V. V.; Dorogan, I. V.; Voloshin, N. A.; Solov'eva, E. V.; Metelitsa, A. V.; Gaeva, E. B.; Aldoshin, S. M.; Minkin, V. I. J. Mol. Struct. 2017, 1145, 55.

[8] Chen, S.; Wang, W. J.; Yan, M. M.; Tu, Q.; Chen, S.-W.; Li, T. B.; Yuan, M.-S.; Wang, J. Y. Sens. Actuators, B 2018, 255, 2086.

[9] Lu, Z. X.; Liu, Y. M.; Lu, S. H.; Li, Y.; Liu, X. L.; Qin, Y.; Zheng, L. Y. RSC Adv. 2018, 8, 19701.

[10] Mehta, P. K.; Oh, E.-T.; Park, H. J.; Lee, K.-H. Sens. Actuators, B 2018, 256, 393.

[11] Chen, M. L.; Lü, X.; Liu, Y. L.; Zhao, Y.; Liu, J.; Wang, P.; Guo, W. Org. Biomol. Chem. 2011, 9, 2345.

[12] Zhang, Y.; Wang, Y.-C.; Mei, J.-G.; Yang, N.-N.; Wang, W.-N.; Ai, S.-F.; Fang, Z.-Q. J. Chem. Res. 2016, 5, 280.

[13] Chen, L.; Park, S. J.; Wu, D.; Kim, H. M.; Yoon, J. Dyes Pigm. 2018, $158,526$.

[14] Shang, Y. T.; Zheng, S. W.; Tsakama, M.; Wang, M.; Chen, W. H. Tetrahedron Lett. 2018, 59, 4003 .

[15] Wang, J. F.; Li, Y. B.; Patel, N. G.; Zhang, G.; Zhou, D. M.; Pang, Y. 
Chem. Commun. 2014, 50, 12258

[16] Pang, Y.; Abeywickrama, C. S. Tetrahedron Lett. 2016, 57, 3518.

[17] Abeywickrama, C. S.; Pang, Y. Tetrahedron Lett. 2017, 58, 1627.

[18] Kwon, J. E.; Lee, S.; You, Y.; Baek, K. H.; Ohkubo, K.; Cho, J.; Fukuzumi, S.; Shin, I.; Park, S. Y.; Nam, W. Adv. Inorg. Chem. Radiochem. 2012, 51, 8760 .

[19] Sadhu, K. K.; Mizukami, S.; Yoshimura, A.; Kikuchi, K. Org. Biomol. Chem. 2013, 11, 563 .

[20] Ma, Q.-J.; Zhang, X.-B.; Zhao, X.-H.; Gong, Y.-J.; Tang, J.; Shen, G.-L.; Yu, R.-Q. Spectrochim. Acta, Part A 2017, 73, 687.

[21] Yang, X. F.; Qin, X. J.; Li, Y. X.; Yan, M. Biosens. Bioelectron. 2018, 121, 62 .

[22] Esteves, C. I. C.; Ferreira, R. C. M.; Costa, S. P. G. Dyes Pigm. 2018, 151, 211

[23] Jiang, K.; Cao, L.; Hao, Z.-F.; Chen, M.-Y.; Cheng, J.-L.; Li, X.; Xiao, P.; Chen, L.; Wang, Z.-Y. Chin. J. Org. Chem. 2017, 37, 2221 (in Chinese).

(蒋凯, 曹梁, 郝志峰, 陈美燕, 程洁金, 李晓, 肖萍, 陈亮, 汪 朝阳, 有机化学, 2017, 37, 2221.)

[24] Chen, L.; Wu, D.; Yoon, J. Sens. Actuators, B 2018, 259, 347.

[25] Li, K.; Feng, Q.; Niu, G. L.; Zhang, W. J.; Li, Y. Y.; Kang, M. M.; Xu, K.; Hou, H. W.; Tang, B. Z. ACS Sens. 2018, 3, 920.

[26] Kaur, I.; Kaur, P.; Singh, K. Sens. Actuators, B 2018, 257, 1083.

[27] Niu, Q. F.; Lan, L. X.; Li, T. D.; Guo, Z. R.; Jiang, T.; Zhao, Z. Y.; Feng, Z. S.; Xi, J. Y. Sens. Actuators, B 2018, 276, 13.

[28] Liu, Y.; Bi, A.Y.; Gao, Tang.; Cao, X. Z.; Gao, F.; Rong, P. F.; Wang, W.; Zeng, W. B. Talanta 2019, 194, 38.

[29] Hou, T. X.; Zhang, K.; Kang, X. X.; Guo, X. L.; Du, L. B.; Chen, X. F.; Yu, L.; Yue, J.; Ge, H. W.; Liu, Y.; Asiri, A. M.; Alamry, K. A.; Yu, H.; Wang, S. H. Talanta 2019, 196, 345.

[30] Nguyen, K. H.; Hao, Y. Q.; Zeng, K.; Fan, S. N.; Li, F.; Yuan, S. K.; Ding, X. J.; Xu, M. T.; Liu, Y. N. Spectrochim. Acta, Part A 2018, 199, 189.

[31] Wang, S. J.; Li, J.; Gao, Y.; Guo, Y. Asian J. Org. Chem. 2018, 7, 563.

[32] Shen, Y. M.; Zhang, X. Y.; Zhang, Y. Y.; Li, H. T.; Dai, L. C.; Peng, X. J.; Peng, Z.; Xie, Y. T. Anal. Chim. Acta 2018, 1014, 71.

[33] Li, J.; Chen, Y. H.; Chen, T.T.; Qiang, J.; Zhang, Z. J.; Wei, T. W.; Zhang, W.; Wang, F.; Chen, X. Q. Sens. Actuators, B 2018, 268, 446.

[34] Feng, G. F.; Luo, X. Y.; Lu, X.; Xie, S. Y.; Deng, L.; Kang, W. Y.; He, F.; Zhang, J. H.; Lei, C. Y.; Lin, B.; Huang, Y.; Nie, Z.; Yao, S. Z. Angew. Chem. 2019, 58, 6590.

[35] Wang, Y. T.; Wang, J. J.; Xian, Q. M. Talanta 2018, 190, 487.

[36] Zhang, J.; Wang, S.; Liu, C. H.; He, G. W.; Peng, T. Y. Chin. J. Chem. 2018, 36, 1179 .

[37] Diao, H. P.; Guo, L. X.; Liu, W.; Feng, L. H. Spectrochim. Acta, Part A 2018, 196, 274.

[38] He, L.; Liu, X. J.; Zhang, Y.; Yang, L.; Fang, Q.; Geng, Y. N.; Chen, W. Q.; Song, X. Z. Sens. Actuators, B 2018, 276, 247.

[39] Hu, Y.; Joung, J. F.; Jeong, J. E.; Jeong, Y.; Woo, H. Y.; She, Y. B.; Park, S.; Yoon, J. Sens. Actuators, B 2019, 280, 298.

[40] Wu, Y.-C.; You, J.-Y.; Guan, L.-T.; Shi, J.; Cao, L.; Wang, Z.-Y. Chin. J. Org. Chem. 2015, 35, 2465 (in Chinese). (吴彦城, 尤嘉宜, 关丽涛, 石杰, 曹梁, 汪朝阳, 有机化学, 2015, 35, 2465.)

[41] Li, G.; Zhang, D. B.; Liu, G.; Pu, S. Z. Tetrahedron Lett. 2016, 57, 5205 .

[42] Liu, L. L.; Dan, F. J.; Liu, W. J.; Lu, X.; Han, Y. L.; Xiao, S. Z.; Lan, H. C. Sens. Actuators, B 2017, 247, 445.

[43] Mashraqui, S. H.; Chandiramani, M.; Ghorpade, S.; Upathayay, J.; Mestri, R.; Chilekar, A. J. Inclusion Phenom. Macrocyclic Chem. 2016, 84, 129.

[44] Wu, Y.-C.; Jiang, K.; Luo, S.-H.; Cao, L.; Wu, H.-Q.; Wang, Z.-Y. Spectrochim. Acta, Part A 2019, 206, 632.

[45] Iniya, M.; Jeyanthi, D.; Krishnaveni, K.; Chellappa, D. J. Lumin. 2015, 157, 383

[46] Li, Z. S.; Li, L. J.; Sun, T. T.; Liu, L. M.; Xie, Z. G. Dyes Pigm.
2016, 128,165 .

[47] Zhu, W. J.; Fang, M.; Zhang, Q.; Xu, Y.; Wu, Z. Y.; Li, C. J. Chin. Chem. Soc. 2018, 65, 597.

[48] Zhao, M. L.; Deng, Z. F.; Tang, J.; Zhou, X. F.; Chen, Z.; Li, X. T.; Yang, L. T.; Ma, L. J. Analyst 2016, 141, 2308.

[49] Bing, Q. J.; Wang, L.; Li, D. L.; Wang, G. Spectrochim. Acta, Part A 2018, 202, 305.

[50] Lu, Z. X.; Liu, Y. M.; Lu, S. H.; Li, Y.; Liu, X. L.; Qin, Y.; Zheng, L. Y. $R S C A d v . \mathbf{2 0 1 8}, 8,19701$.

[51] Krishnaveni, K.; Iniya, M.; Jeyanthi, D.; Siva, A.; Chellappa, D. Spectrochim. Acta, Part A 2018, 205, 557.

[52] Sen Gupta, A.; Garg, A.; Paul, K.; Luxami, V. J. Lumin. 2016, 173, 16.

[53] Ge, Y. Q.; Liu, A. K.; Ji, R. X.; Shen, S. L.; Cao, X. Q. Sens. Actuators, $B$ 2017, 251, 410.

[54] Goswami, S.; Maity, S.; Maity, A. C.; Maity, A. K.; Das, A. K.; Saha, P. RSC Adv. 2014, 4, 6300.

[55] Chen, C. J.; Liu, H. Y.; Zhang, B.; Wang, Y. W.; Cai, K.; Tan, Y.; Gao, C. M.; Liu, H. X.; Tan, C. Y.; Jiang, Y. Y. Tetrahedron 2016, 72,3980 .

[56] Ta, S.; Das, S.; Ghosh, M.; Banerjee, M.; Hira, S. K.; Manna, P. P.; Das, D. Spectrochim. Acta, Part A 2019, 209, 170.

[57] Paul, A.; Anbu, S.; Sharma, G.; Kuznetsov, M. L.; da Silva, M. F. C. G.; Koch, B.; Pombeiro, A. J. L. Dalton Trans. 2015, 44, 16953.

[58] Dhaka, G.; Kaur, N.; Singh, J. Inorg. Chem. Commun. 2016, 72, 57.

[59] Gao, Y. L.; Zhang, C.; Peng, S. W.; Chen, H. Y. Sens. Actuators, B 2017, 238, 455.

[60] Bieleszova, K.; Parizkova, B.; Kubes, M.; Husickova, A.; Kubala, M.; Ma, Q.; Sedlarova, M.; Robert, S.; Dolezal, K.; Strnad, M.; Novak, O.; Zukauskaite, A. New Biotechnol. 2019, 48, 44.

[61] More, G. V.; Bhanage, B. M. Catal. Sci. Technol. 2015, 5, 1514.

[62] Samanta, S.; Ray, T.; Haque, F.; Das, G. J. Lumin. 2016, 171, 13.

[63] Fan, L.; Gao, S. Q.; Li, Z. B.; Niu, W. F.; Zhang, W. J.; Shuang, S. M.; Dong, C. Sens. Actuators, B 2015, 221, 1069

[64] Joshi, S.; Kumari, S.; Sarmah, A.; Pant, D. D.; Sakhuja, R. J. Mol. Liq. 2017, 248, 668 .

[65] Lin, W. C.; Hu, J. W.; Chen, K. Y. Anal. Chim. Acta 2015, 893, 91.

[66] Shan, Y. Y.; Wu, Q. Q.; Sun, N.; Sun, Y. H.; Cao, D. X.; Liu, Z. Q.; Guan, R. F.; Xu, Y. X.; Yu, X. Y. Mater. Chem. Phys. 2017, 186, 295

[67] Tang, Y.; Jiang, G. F. Tetrahedron Lett. 2017, 58, 2846.

[68] Tumay, S. O.; Okutan, E.; Sengul, I. F.; Ozcan, E.; Kandemir, H.; Doruk, T.; Cetin, M.; Cosut, B. Polyhedron 2016, 117, 161.

[69] Xu, T.; Duan, H. D.; Wang, X. J.; Meng, X.; Bu, J. Spectrochim. Acta, Part A 2015, 138, 603.

[70] Jeyanthi, D.; Iniya, M.; Krishnaveni, K.; Chellappa, D. Spectrochim. Acta, Part A 2015, 136, 1269

[71] Lv, Z. X.; Sun, Z. W.; Song, C. H.; Lu, S. M.; Chen, G.; You, J. M. Talanta 2016, 161, 228.

[72] Huang, Y. L.; Zhou, Q.; Feng, Y.; Zhang, W.; Fang, G. S.; Fang, M.; Chen, M.; Xu, C. Z.; Meng, X. M. Chem. Commun. 2018, 54, 10495

[73] Ning, P.; Hou, L. L.; Feng, Y.; Xu, G. Y.; Bai, Y. Y.; Yu, H. Z.; Meng, X. M. Chem. Commun. 2019, 55, 1782.

[74] Feng, Y.; Li, S. Q.; Li, D. X.; Wang, Q.; Ning, P.; Chen, M.; Tian, X. H.; Wang, X. Sens. Actuators, B 2018, 254, 282.

[75] Shi, G.; Shahid, M. A.; Yousuf, M.; Mahmood, F.; Rasheed, L.; Bielawski, C. W.; Kim, K. S. Chem. Commun. 2019, 55, 1470

[76] Lin, D. Y.; Fei, X. N.; Li, R.; Gu, Y. C.; Tang, Y. L.; Zhou, J. G.; Zhang, B. L. J. Fluoresc. 2016, 26, 1511.

[77] Patra, L.; Aich, K.; Gharami, S.; Mondal, T. K. J. Lumin. 2018, 201, 419.

[78] Bi, K. Y.; Tan, R.; Hao, R. T.; Miao, L. X.; He, Y. Q.; Wu, X. H.; Zhang, J. F.; Xu, R. Chin. Chem. Lett. 2019, 30, 545.

[79] Kandemir, H.; Kocak, A.; Tumay, S. O.; Cosut, B.; Zorlu, Y.; Sengul, I. F. Turk. J. Chem. 2018, 42, 221.

[80] Sanchez, G.; Espinosa, A.; Curiel, D.; Tarraga, A.; Molina, P. J. Org. Chem. 2013, 78, 9725. 
[81] Yang, L. L.; Wang, J. P.; Yang, L.; Zhang, C.; Zhang, R. L.; Zhang, Z. P.; Liu, B. H.; Jiang, C. L. RSC Adv. 2016, 6, 56384.

[82] Wang, W. D.; Hu, Y.; Hu, S. L. Inorg. Chim. Acta 2018, 477, 206.

[83] Zhou, F. F.; Wang, H. Q.; Liu, P. Y.; Hu, Q. H.; Wang, Y. Y.; Liu, C.; Hu, J. K. Spectrochim. Acta, Part A 2018, 190, 104.

[84] Liu, X. J.; Su, Y. A.; Tian, H. H.; Yang, L.; Zhang, H. Y.; Song, X. Z.; Foley, J. W. Anal. Chem. 2017, 89, 7038.

[85] Wu, G. F.; Li, M. X.; Zhu, J. J.; Lai, K. W. C.; Tong, Q. X.; Lu, F. RSC Adv. 2016, 6, 100696

[86] Gupta, R. C.; Razi, S. S.; Ali, R.; Dwivedi, S. K.; Srivastava, P.; Singh, P.; Koch, B.; Mishra, H.; Misra, A. Sens. Actuators, B 2017, 251,729 .

[87] Wang, L. F.; Qian, Y. J. Photochem. Photobiol., A 2019, 372, 122.

[88] Dai, Y. P.; Yao, K.; Fu, J. X.; Xue, K.; Yang, L.; Xu, K. X. Sens. Actuators, B 2017, 251, 877.

[89] Obali, A. Y.; Yilmaz, M. S.; Ucan, H. I. J. Mol. Struct. 2017, 1145, 192.

[90] Liu, H. Y.; Tan, Y.; Dai, Q. Z.; Liang, H. D.; Song, J.; Qu, J. L.; Wong, W. Y. Dyes Pigm. 2018, 158.

[91] Luo, Z. L.; Yin, K.; Yu, Z.; Chen, M. X.; Li, Y.; Ren, J. Spectrochim. Acta, Part A 2016, 169, 38.

[92] Li, B.; Tian, J. F.; Zhang, D.; Tian, F. L. Luminescence 2017, 32, 1567.

[93] Marimuthu, P.; Ramu, A. Sens. Actuators, B 2018, 266, 384.

[94] Murugan, A. S.; Vidhyalakshmi, N.; Ramesh, U.; Annaraj, J. Sens. Actuators, B 2018, 274, 22.

[95] Adhikari, S.; Mandal, S.; Ghosh, A.; Guria, S.; Pal, A.; Adhikary, A.; Das, D. J. Photochem. Photobiol., A 2018, 360, 26.

[96] Han, J.; Tang, X.; Wang, Y.; Liu, R. J.; Wang, L.; Ni, L. Spectrochim. Acta, Part A 2018, 205, 597.

[97] Manikandan, I.; Chang, C. H.; Chen, C. L.; Sathish, V.; Li, W. S.; Malathi, M. Spectrochim. Acta, Part A 2017, 182, 58

[98] Deng, Y.; Niu, W. P.; Wang, Z. J.; Feng, L. H. Sens. Actuators, B 2017, 238, 613.

[99] Zhao, Q.; Liu, X. M.; Li, H. R.; Zhang, Y. H.; Bu, X. H. Dalton Trans. 2016, 45, 10836.

[100] Cui, M. M.; Li, W. T.; Wang, L. Y.; Gong, L. S.; Tang, H.; Cao, D. R. J. Mater. Chem. C 2019, 7, 3779.

[101] Weldekirstos, H. D.; Kuo, M. C.; Li, S. R.; Su, W. L.; Desta, M. A.;
Wu, W. T.; Kuo, C. H.; Sun, S. S. Dyes Pigm. 2019, 163, 761.

[102] Chen, H. Y.; Zhou, Z. L.; Lu, Q. J.; Wu, C. Y.; Liu, M. L.; Zhang, Y. Y.; Yao, S. Z. Anal. Chim. Acta 2019, 1051, 160.

[103] More, Y. W.; Padghan, S. D.; Bhosale, R. S.; Pawar, R. P.; Puyad, A. L.; Bhosale, S. V.; Bhosale, S. V. Sensors 2018, 18, 3433.

[104] Mahanta, S. P.; Panda, P. K. J. Chem. Sci. 2017, 129, 647.

[105] Han, H.; Liu, Y. R.; Dong, C.; Han, X. E. J. Lumin. 2017, 183, 513.

[106] Ishtiaq, M.; Munir, I.; Al-Rashida, M.; Maria.; Ayub, K.; Iqbal, J.; Ludwig, R.; Khan, K. M.; Ali, S. A.; Hameed, A. RSC Adv. 2016, 6, 64009 .

[107] Feng, L.; Shi, W.; Ma, J. C.; Chen, Y. B.; Kui, F.; Hui, Y. H.; Xie, Z. F. Sens. Actuators, B 2016, 237, 563.

[108] Che, C. L.; Chen, X. Z.; Wang, H. M.; Li, J. Q.; Xiao, Y. M.; Fu, B.; Qin, Z. H. New J. Chem. 2018, 42, 12773.

[109] Shi, W.; Lei, Y. L.; Hui, Y. H.; Mi, H. Y.; Ma, F. D.; Tian, Y.; Xie, Z. F. New J. Chem. 2014, 38, 4730.

[110] Cui, W.; Wang, L. Y.; Xiang, G.; Zhou, L. X.; An, X. N.; Cao, D. R. Sens. Actuators, B 2015, 207, 281.

[111] Zhu, L. M.; Yang, J.; Wang, Q. S.; Zeng, L. T. J. Lumin. 2014, 148, 161.

[112] Zhang, H. L.; Wei, T. B.; Li, W. T.; Qu, W. J.; Leng, Y. L.; Zhang, J. H.; Lin, Q.; Zhang, Y. M.; Yao, H. Spectrochim. Acta, Part A 2017, $175,117$.

[113] de Moliner, F.; King, A.; Dias, G. G.; de Lima, G. F.; de Simone, C. A.; da Silva, E. N.; Vendrell, M. Front. Chem. 2018, 6, 339.

[114] Shi, B. B.; Zhang, Y. M.; Wei, T. B.; Lin, Q.; Yao, H.; Zhang, P.; You, X. M. Sens. Actuators, B 2014, 190, 555.

[115] Li, W. T.; Wu, G. Y.; Qu, W. J.; Li, Q.; Lou, J. C.; Lin, Q.; Yao, H.; Zhang, Y. M.; Wei, T. B. Sens. Actuators, B 2017, 239, 671.

[116] Gao, G. Y.; Qu, W. J.; Shi, B. B.; Lin, Q.; Yao, H.; Zhang, Y. M.; Chang, J.; Cai, Y.; Wei, T. B. Sens. Actuators, B 2015, 213, 501.

[117] Aggarwal, K.; Khurana, J. M. J. Photochem. Photobiol., A 2015, $307,23$.

[118] Aggarwal, K.; Khurana, J. M. J. Lumin. 2015, 167, 146.

[119] Jiang, K.; Luo, S.-H.; Pang, C.-M.; Wang, B.-W.; Wu, H.-Q.; Wang Z.-Y. Dyes Pigm. 2019, 162, 367.

[120] Jiang, K.; Chen, S.-H.; Luo, S.-H.; Pang, C.-M.; Wu, X.-Y.; Wang Z.-Y. Dyes Pigm. 2019, 167, 164. 